\title{
CULTURAL DIFFERENCES IN MEMORY FOR FACES AND ASSOCIATED PERCEPTUAL VERSUS SOCIAL CONTEXTS
}

\author{
by \\ Lingqian Li
}

B.Sc. (Hons) Psychology and Human Biology, University of Toronto, 2011

\author{
A thesis \\ presented to Ryerson University \\ in partial fulfilment of the \\ requirement for the degree of \\ Master of Arts \\ in the Program of \\ Psychology \\ Toronto, Ontario, Canada, 2014 \\ CLingqian Li 2014
}




\section{AUTHOR'S DECLARATION FOR ELECTRONIC SUBMISSION OF A THESIS}

I hereby declare that I am the sole author of this thesis. This is a true copy of the thesis, including any required final revisions, as accepted by my examiners.

I authorize Ryerson University to lend this thesis to other institutions or individuals for the purpose of scholarly research.

I further authorize Ryerson University to reproduce this thesis by photocopying or by other means, in total or in part, at the request of other institutions or individuals for the purpose of scholarly research.

I understand that my thesis may be made electronically available to the public. 
Cultural Differences in Memory for Faces and Associated Perceptual and Social Contexts Lingqian Li, B.Sc. (Hons), Master of Arts, 2014

Psychological Science, Ryerson University

\begin{abstract}
The current study investigates cultural differences in memory for faces and their associated contexts between East Asian and Western cultures. Thirty-six Caucasian Canadian (age range $=17-30, M=21.00, S D=3.94)$ and 35 native Chinese (age range $=$ $19-27, M=22.06, S D=2.33$ ) young adults participated in the study. At encoding, participants subjectively rated face stimuli according to the cue words within social ("FRIENDLY" vs. "SUCCESS") and perceptual ("HEIGHT" vs. "WEIGHT") contexts. At test, participants performed a context recognition task to identify whether the test stimulus was a new face or an old face associated with a specific context cue word. Results did not show any cultural difference in memory. However, both cultures showed better recognition in the social context than in the perceptual context condition. In addition, the other race effect was observed in context memory for Canadian, but not Chinese participants.
\end{abstract}




\section{Acknowledgements}

I would like to express my deepest appreciation to my supervisor and committee chair, Dr. Lixia Yang, for her patience and continuous guidance throughout the two years. Without her guidance and persistent help this thesis would never have taken shape. I am also grateful to Dr. Julia Spaniol and Dr. Margaret Moulson for their insightful comments and suggestions as my examining committee.

My thanks also go out to my collaborator Weicong Ren and Dr. Juan Li. Their support made the Chinese sample data collection such a successful and smooth process. I would also like to thank the diligent research assistants involved in the data collection and entry processes for this project: Elena Anguelova, Kathleen Lyons, Malika Ibragimova and Yuan Zhao.

In addition, I am grateful to my fellow graduate students in the lab, Andrea Wilkinson, Linda Truong, Brenda Wong, Sara Gallant and Dana Greenbaum. Their support and companion made my graduate school experience much more enjoyable and much less stressful.

Last but not least, I would like to thank my friends and family. It is their understanding and encouragement that made me who I am today.

This project is supported by the Canadian Institute of Health Research Grant (CIHR; CCI-102930) awarded to Dr. Lixia Yang. 


\section{Table of Contents}

Abstract........................................................................................................................ii

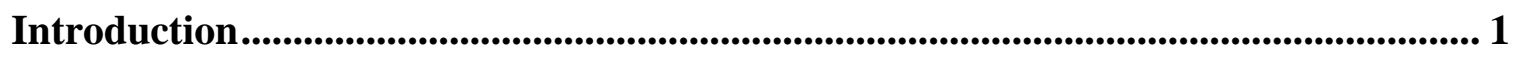

Cultural Differences in Focal and Contextual Information Processing .......................... 3

Cultural Differences in Item-Context Association Memory ………………………....... 5

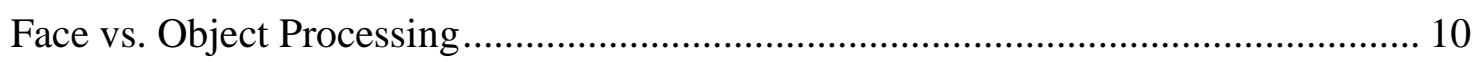

Cultural Differences in Face Processing and Recognition.............................................. 12

The Other Race Effect in Face Processing and Recognition ......................................... 13

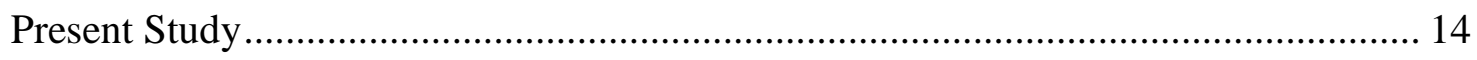

Method ................................................................................................................................................ 18

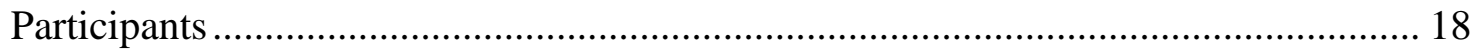

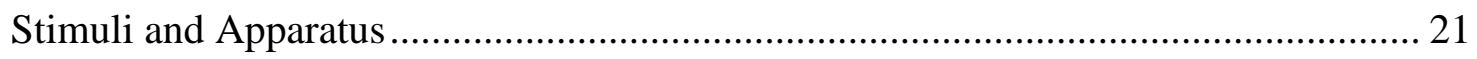

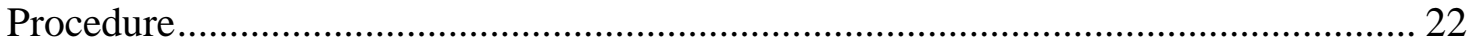

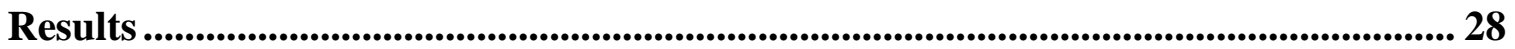

Statistical Analyses .......................................................................................... 28

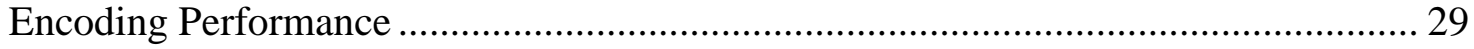

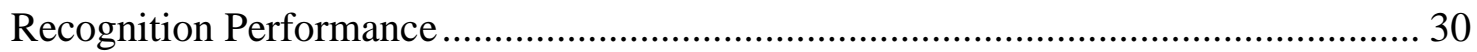

The Other Race Effect on Memory Performance........................................................... 32

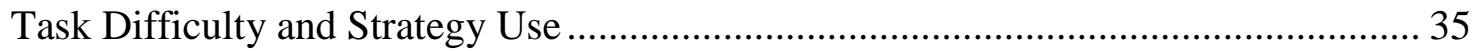

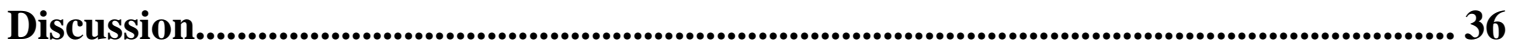

The Unique Mechanisms Underlying Face Processing................................................ 36

Perceptual vs. Social Context in Face Processing .......................................................... 40

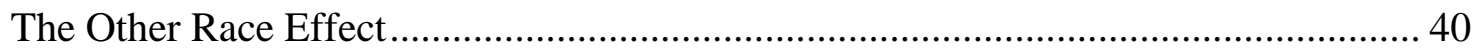

Limitations and Future Directions..................................................................... 42

Conclusion and Implications.................................................................................................. 43

Appendix I: Participant Consent Forms (Community Recruitment) ........................... 45

Appendix II: Participant Consent Forms (SONA Recruitment)................................... 48

Appendix III: Participant Debriefing Sheet ............................................................................. 51

Appendix IV: Photo Difficulty Rating Questionnaire ......................................................... 53

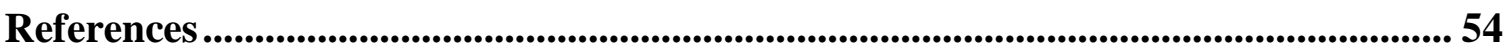




\section{List of Tables}

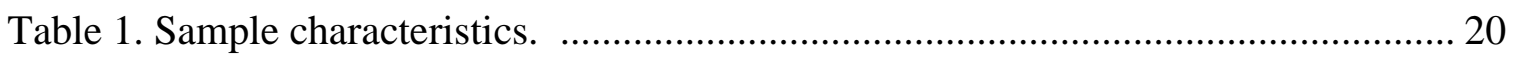

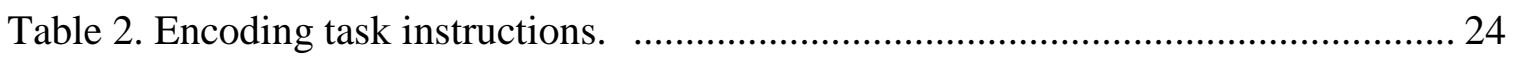

Table 3. Group means in encoding RTs and rating scores. ....................................... 29

Table 4. Group means in memory scores during recognition ..................................... 30

\section{List of Figures}

Figure 1. Example of the experimental paradigm in the social context block................. 26

Figure 2. Group means of recognition RTs in perceptual and social context blocks ....... 32

Figure 3. Group mean item memory scores across context blocks and races................. 33

Figure 4. Group mean context memory scores across context blocks and races .............. 34 
Cultural Differences in Memory for Faces and Associated Perceptual and Social Contexts

Since the burgeoning of "cognitive revolution" in psychology, there has been a predominant belief that most basic human cognitive functions, such as language, attention and memory processes, are innate and universal for all human beings (e.g., Chomsky, 2002). A popular illustration of this belief at the time was the brain-computer analogy, in which the human mind is compared to the processing units of a computer (Block, 1995). According to this analogy, all human brains, like the hard-wired computers, will always operate on the incoming information under identical mechanisms. However, emerging research evidence has challenged this universality assumption, by demonstrating cultural differences in various cognitive domains, such as attention at the early stages of information processing (for extensive reviews, see Buchtel \& Norenzayan, 2009; Han et al., 2013; Nisbett \& Norenzayan, 2002; Norenzayan \& Heine, 2005; Yama, Nishioka, Horishita, Kawasaki, \& Taniguchi, 2007).

In particular, studies found that individuals from Western cultures (e.g., Western Europe and North America) tend to think analytically - they focus on feature-specific and rule-based aspects of information. Conversely, East Asians (e.g., Chinese, Korean, and Japanese) have a higher tendency to think holistically - they pay more attention to contextual and thematically associated information (Nisbett \& Miyamoto, 2005). In an effort to determine one' habitual thinking style, Choi and colleagues (2007) developed the Analysis-Holism Scale (AHS). This scale measures to what extent one identifies with statements such as "Everything in the universe is somehow related to each other" and "It is more important to pay attention to the whole context rather than the details". Individuals receiving high scores on this scale are expected to engage in thinking styles 
that are more holistic than those with low scores. As expected, East Asian participants were found to score higher on this scale than Western participants (Experiment 3). In addition, the AHS score were strongly correlated with the tendency one prioritize contextual and associative information in cognitive tasks (Experiments 5 and 6).

It seems that the differential processing styles between East and West are at least partially driven by their cultural and philosophical roots. In ancient Greek philosophy, every object or person is considered as an separate and distinctive entity from each other; whereas in Confucianism and Taoism, the two predominant schools of thoughts that influenced ancient East Asia, the universe is defined as a network of interdependent substances and overlapping networks of energy (Hansen, 1983). Meanwhile, the two cultures differ vastly in social structures. Specifically, individualism is predominant in Western societies, in which people prioritize independent and personal goals without being overly constrained by the demand of the society. In contrast, Eastern societies are most collectivism-based, in which members are dependent on each other and work as a whole to serve the interest of the society (Triandis, 1995).

Drawing from the these established cultural differences in historically and socially adopted values between the East and the West, Nisbett and colleagues (Nisbett \& Masuda, 2003; Nisbett \& Miyamoto, 2005; Nisbett, Peng, Choi, \& Norenzayan, 2001) proposed a social-cognitive framework that categorizes the distinct thought processes people from different cultural backgrounds tend to engage. This framework provides valuable insight in the research of cultural differences in human cognition. According to this framework, the specific social value a person holds will preferentially direct their attention to some 
aspects of the information over others, which subsequently will influences the one's

perception of the reality (Nisbett et al., 2001).

\section{Cultural Differences in Focal and Contextual Information Processing}

Inspired by this social-cognitive framework, a growing number of studies have started to investigate in what aspects, and to what extent, cultural backgrounds can shape information processing styles. One particular area that gained popularity during the last decade is the investigation of cultural differences in the processing of focal and contextual information (e.g., Ko, Lee, Yoon, Kwon, \& Mather, 2011; Masuda \& Nisbett, 2006; Masuda et al., 2008; Nisbett et al., 2001). Emerging evidence suggests that people from East Asian cultures generally incorporate more contextual elements and item-context relationships during information processing, whereas people from Western cultures tend to de-contextualize the information and process them systematically (Masuda \& Nisbett, 2001). For instance, in the study conducted by Masuda and Nisbett (2006), American and Japanese participants were instructed to study still photos (experiment 1) and short animated movie clips (experiment 2 and 3) and later report if any changes were made when they were presented again. They found that Japanese participants, compared to American participants, were faster to detect changes made to the context than to the focal object. Conversely, American participants were faster to identify changes made to the focal objects than to the context, and detected more changes made to the focal objects than did the Japanese participants.

Similar cultural effects on context processing patterns were observed at behavioural and perceptual levels. In an eye-tracking study, Chua and colleagues (2005) tracked participants' eye movement patterns during an active scene viewing task. They 
found that American individuals fixated more on the focal objects of the scene, and attended to the focal objects earlier in the process. In contrast, Chinese individuals preferentially made more eye movements to the backgrounds of the scenes, suggesting their visual attention is more directed to the context and that they view the scenes more holistically. Similarly, studies have suggested that East Asians attended to a broader perceptual field and were more sensitive to contextual information during facial expression processing tasks (Ko et al., 2011; Masuda et al., 2008). In addition, Ji and colleagues (2000) found that Chinese participants' had greater difficulty differentiating the focal targets from the field compared to their American counterparts.

Several neuroimaging studies further supported the notion of cultural influences on information processing, demonstrating culture-specific patterns of neural activation during different cognitive tasks (e.g., Gutchess, Welsh, Boduroglu, \& Park, 2006; Hedden, Ketay, Aron, Markus, \& Gabrieli, 2008; Jenkins, Yang, Goh, Hong, \& Park, 2010). Park and Huang (2010) suggested that prolonged exposure and practice of culturally preferred strategies may influence the neural structure and functioning of human brain. For instance in an fMRI study, Gutchess and colleagues (2006) found that Westerners activated more object-processing regions (i.e., left superior parietal, right superior temporal/ supra-marginal gyrus, and left middle temporal lobe) in the brain during complex scene viewing than did East Asians. In addition, Chinese participants showed an increase in object processing activation in perceptual areas (i.e., lateral occipital regions) during the passive viewing of incongruent scenes compared to congruent scenes. In contrast, American participants did not show this biased attention towards objects in the incongruent condition (Jenkins et al., 2010). These findings 
suggest that Chinese participants were more sensitive to incongruity between the focal object and the peripheral scene than American participants. Furthermore, in a frame-line test for which participants need to make absolute (ignore the frame surrounding the line) or relative (incorporate the size of the surrounding frame) judgements of line length, Hedden and colleagues (2008) showed increased attentional engagement (activation in frontal and parietal regions) for American during their culturally non-preferred relative judgement task and for Chinese during their culturally non-preferred absolute judgment. This finding further supports the notion that Westerners and East Asians have distinct information processing styles, and their brains demand more cognitive resources when the task instruction does not align with their styles.

In summary, both behavioural and neuroimaging studies revealed convergent evidence for cultural differences in information processing styles between the East and the West. Individuals in East Asian cultures commonly adopt a context-integrated holistic processing style whereas those in Western European cultures are more likely to engage a feature-based analytic processing of the focal items.

\section{Cultural Differences in Item-Context Association Memory}

The cultural differences observed in context processing have led to the exploration of whether these differences in perception and cognition could be extended to memory functions. If people from different cultural backgrounds utilize distinct information processing styles as previously described, then there could also be cultural differences in terms of what aspects of information can be better preserved in memory and more accessible during retrieval. Current literature shows mixed findings on culture differences in item-context association and memory performance. 
Some studies suggest that East Asians may be able to associate objects with the corresponding context more readily than Westerners. For instance, in Masuda and Nisbett (2001) Japanese and American participants were instructed to study animated vignettes of underwater scenes, and later recall the scene by describing what they remembered. American participants' responses primarily focused on the focal objects, in this case some prominent fish at the center of the scene; in contrast Japanese participants' responses contained more contextual details (e.g., seaweeds in the background, the bubbles in the water, etc.). In the following surprise recognition task, participants were asked to identify whether presented object was seen in the vignettes. Interestingly, Japanese participants' recognition memory for the focal objects suffered when the associated backgrounds were removed or replaced by novel scenes; however American participants' object memory was unaffected by this manipulation. Similar cultural differences have been observed between American and Chinese participants (Chua et al., 2005). In this study participants were instructed to study computer-generated images with focal objects superimposed onto background scenes, and later identify whether presented objects were studied or new. They found the Chinese participants were less accurate in correctly recognizing objects when they were paired with new backgrounds. Both studies demonstrated that individuals from East Asian cultures seem to bind the contextual information, in these two cases background scenes, with the focal item more readily than Western individuals.

At the same time, other studies did not detect this cultural difference in item-context associative memory. For example, using an fMRI-adaptation paradigm, Goh et al. (2007) monitored East Asian and Western participants' (both younger and older 
adults) blood oxygen level dependent (BOLD) responses in the brain when they passively view repeated presentation of pictures with different combinations of repeated or novel focal objects and backgrounds. Specifically, the item-context binding was indexed by neural adaptation (i.e., reduced neural activity) to repeated object-context pairs within a trial, in comparison with all the other types of combinations. The greater the adaptation, the stronger the item-context binding a person holds in mind. Results did not show any culture difference in the neural adaptation in response to item-context binding, suggesting that the two culture groups were able to bind the items and contexts equally well. Moreover, in Chua, Chen and Park (2006), Chinese and American participants watched videos with several factual statements introduced by different speakers. In a subsequent incidental memory task, participants were asked to identify the source (i.e., speaker) for each spoken statement. The result showed no cultural difference in source memory performance. They concluded that it was likely that people from different cultures operate with the same universal basic source memory mechanisms.

Upon closer examination, it appears that in both Goh et al. (2007) and Chua et al. (2006), the associations between item and context were arbitrarily defined and passively presented. This means that only incidental memory for abstract associations was involved in these studies, which probably does not generalize to real life scenarios. In a recent publication, Yang and colleagues (2013) demonstrated that Chinese participants outperformed their Canadian counterparts in an item-context binding memory task that promoted integration of presented items within socially meaningful contexts. In this study, participants were instructed to actively examine object images by rating the objects in socially meaningful contexts based on the provided cue words. In the "independent" 
block, participants evaluated the objects either in terms of their meaningfulness for them to lead an independent life in a new city or in terms of the typicality of the objects in everyday life. Whereas in the "relational" block, participants evaluated the objects either on their usefulness for them to get along with other people in a new city or on the typicality of the objects in everyday life. The results showed that, relative to the Canadian participants, the Chinese were better at recalling the particular context cues associated with studied items. The authors speculated that these socially meaningful item-context association instructions could have activated more culturally salient knowledge and experiences for the Chinese participants than Canadian participants, which lead to their enhanced memory for item-context associations in the recognition task. Therefore, it is possible that cultural differences in item-context binding performance could only exit when the association between the item and the context is meaningful and socially salient.

At the same time, it should be noted that the effortful rating process engaged at encoding could have also contributed to the East Asians' advantage in memory for socially meaningful contexts. As illustrated in Hedden et al. (2008), although no cultural differences was found in the behavioural data, individuals showed increased activation in brain regions that governs attention, cognitive control and memory (i.e., frontal, parietal and temporal regions) when performing their culturally non-preferred tasks. This finding suggests that these culturally less familiar tasks may be more difficulty and resource-demanding. As previously reviewed, individuals from East Asian cultures tend to process information holistically by associating the item to the context (Nisbett et al., 2001). Therefore, when tasks such as that in Yang et al. (2013) require participants to 
integrate object information within the various contexts in a scenario, it may be less cognitively demanding for the Chinese participants.

Overall, literature on cultural differences in item-context association memory has shown mixed findings. Despite the recent evidence of cultural differences in memory for socially meaningful item-context associations (Yang et al., 2013), it remains unclear whether this memory advantage in Chinese participants was driven by the socially salient context instructions, by the processes engaged at encoding, or the combination of the two. To disentangle these factors, the present study adopted a similar item-context rating procedure as Yang et al. (2013), but with the addition of a second non-social perceptual context condition. If socially salient context was the main cause of better item-context association observed in the previous study for Chinese participants, then this memory advantage should be reduced or even eliminated in the perceptual context task.

Alternatively, if the item-context rating instruction was the main cause regardless of the social salience, cultural effects should be equivalent across the social and perceptual context conditions.

To promote processing of item-context associations and for the convenience of matching social and perceptual conditions, human faces were chosen as the test stimuli in the current study. This also allows for the examination of whether the cultural effects found in object-context memory binding could be generalized to face-context binding. This examination is ecologically valid given that successful recognition of faces and the associated context/situations (e.g., threating or welcoming context) has implications in real life. However, before proceeding to investigate cultural differences in face-context 
association performance, it is important to review the potential differences between the processing mechanism of objects and human faces.

\section{Face vs. Object Processing}

Many theories developed for object processing may not be directly applicable to face processing. For one, the processing of face information carries greater social salience than the processing of other types of objects. As a social species, human beings utilize face perception strategies to navigate social interactions with other individuals on a regular basis. Study found that the viewing of a human face stimuli could trigger automatic inferences and social judgement, as reflected by brain activation patterns, without the explicit instruction to do so (Todorov, Mende-Siedlecki, \& Dotsch, 2013). In support of this, research has linked the impairment of social skills in patients with autism spectrum disorder with their inability to interpret facial cues during development (Schultz, 2005).

Furthermore, it has been suggested that face processing, compared to object processing, is more dependent on one's ability to utilize the global or configure representations of faces than to inspect the features separately (Tanaka \& Farah, 2003). In a classical study, Tanaka and Farah (1993) had participants identify partial features of previously studied stimuli (i.e., normal faces, scrambled faces, inverted faces, and houses) either in isolation or within the context of the whole face. Results showed that participants were better at recognizing the face features when they were presented within the whole face than when they were presented in isolation, yet this context benefit was not found in any other types of stimuli. This specialized holistic processing mechanism enabled people to identify and discriminate individuals among highly similar visual 
information in the faces. However, it is important to understand that the term "holistic processing" used in face recognition literature does not carry the same meaning as when it is used in cross-cultural cognitive research. In face processing, holistic processing primarily refers to the holistic integration of internal features and spatial configuration within the item (face stimuli as a whole). In cross-cultural cognitive studies (e.g., Yang et al., 2013), holistic processing mainly refers to the style of processing items/objects in association with meaningful external information (i.e., background or context).

In addition to the aforementioned processing differences, face and object recognition may also rely on different memory strategies. Most non-face experimental stimuli used in recognition paradigms (e.g., words, common objects, and animals) can be discriminated based on the rich conceptual or semantic representations (such as name, category, meaning and/or functions) associated with the item. However, given that most human faces share the same configuration (i.e., eyes are positioned to both sides of the face above the nose, the mouth is oriented at the bottom, etc.), it is hard to extract distinct semantic information about the person (i.e., gender, race, emotional state, social role etc.) without inspecting the facial details visually. So relative to object recognition that is likely aided by the semantic/functional information associated with a specific object, face recognition primarily relies on perceptual information derived from examination of facial features and their spatial configurations. Richler, Cheung and Gauthier (2011) found that individual differences in holistic processing abilities could predict one's ability to correctly recognize human faces in memory tasks. In addition, this specific holistic processing behavior has been associated with better recognition independent of general cognitive abilities such as intelligence or attention (Wang, Li, Fang, Tian, \& Liu, 2012). 
Taken together, people seem to rely on different processing strategies during the cognition of faces from when they process common objects. The differences are found not only in early encoding stage, but also in subsequent recognition processes. Overall, face processing/recognition tends to rely on holistic processing of features within the face whereas object processing/recognition could recruit more conceptual/semantic knowledge of the object such as its meaning and functions. Considering these differences, it is speculated that perceptual feature-based face processing might reflect more fundamental cognitive mechanics that is universal across culture groups. In contrast, the conceptual object processing may involve more knowledge-dependent cognitive pragmatics which may vary across cultures (Baltes, 1993). Following this reasoning, the cultural differences reported in object processing may not generalize to face processing and recognition.

\section{Cultural Differences in Face Processing and Recognition}

The mechanisms for face processing are argued to be more holistic than object processing in nature, even for Western individuals who generally prefer the analytical, feature-driven information processing style. Cross-culture studies have compared face perception and recognition performance between East Asian and Western cultures, and showed inconsistent results for the two domains.

Studies have demonstrated cultural differences in processing styles during face perception. In Miyamoto, Yoshikawa, and Kitayama (2011), Japanese and Caucasian American participants were asked to choose between two morphed faces as the best representation for a set of previously studied exemplar faces. Results showed that Japanese participants were more likely to select morphed faces that had similar spatial 
configuration as the studied faces, while the Caucasian American participants were more likely to choose faces that had higher resemblance of facial features. Eye-tracking evidences revealed cultural difference in face processing styles as well. In Blais, Jack, Scheepers, Fiset, \& Caldara (2008), despite showing culturally comparable recognition performance in the memory task, the two culture groups demonstrated distinct viewing patterns. The Caucasian participants allocated most attention to specific facial features such as the eyes and the mouth throughout the task, whereas the East Asians were more fixated to the central region (nose) and attended to the facial features evenly.

Despite these cultural differences demonstrated in face processing styles, none of these studies reported group difference in recognition of face stimuli. It seems that the cultural preference in face processing styles does not necessarily affect how well facial information is stored and retrieved. In fact, Caldara, Zhou and Miellet (2010) suggested that face recognition did not differ across the two cultures, instead people rely on the same facial information (the eyes and the mouth) to recognize faces.

\section{The Other Race Effect in Face Processing and Recognition}

Meanwhile, face processing research shows that the ability to learn and recognize faces seemed to be subject to the matching of race between the observer and the to-be-examined face. The other race effect, as repetitively demonstrated in the face recognition studies, suggests that people experience greater difficulty recognizing faces that do not belong to one's racial group (for review, see Meissner \& Brigham, 2001). Explanations for this effect range from different levels of social exposure, perceptual expertise, different representational models, to different underlying processing mechanisms (Young, Hugenberg, Bernstein, \& Sacco, 2012). Furthermore, the other race 
effect has been demonstrated in both East Asian and Western cultures. For instance, as demonstrated in Rhodes, Hayward and Winkler (2006), both Caucasian and Chinese participants were more accurate at detecting changes made to studied faces of their own race compared to ones from the other race.

However, the other-race effect can also manifest itself differently between East Asian and Western cultures. Tanaka, Kiefer and Bukach (2004) found different degrees of holistic processing (significantly better recognition for whole face stimuli than partial features) when Western and Asian participants were asked to identify Caucasian and East Asian faces in the short-delay face recognition task. In particular, they found that compared to East Asian participants who demonstrated equivalent levels of holistic processing style for both same-race and other-race faces, the Western participants only demonstrated the holistic processing style for Caucasian faces but not for East Asian faces.

To this point, most work on face processing has exclusively focused on the processing and recognition of face stimuli per se. Little is known about the processing mechanism and memory performance for the specific contextual information associated with the face during encoding. To fill this gap, the current study examined potential cultural differences in face-context processing behavior and memory performance between the West and the East.

\section{Present Study}

The present study compared memory for faces and face-context associations between Chinese and Canadian young adults with a similar experimental paradigm as that in Yang et al. (2013). Specifically, the active and elaborative rating encoding was 
adopted in which participants rate target stimuli from different perspectives based on a provided context cue. Two major modifications were made in addition to the change of stimuli type from line-drawing objects to human faces.

First, a perceptual context condition was introduced in addition to the socially meaningful context condition. In the social context condition, participants were instructed to evaluate face stimuli in terms of how friendly or successful a person appeared relative to an average person; while in the perceptual context condition, participant were instructed to estimate the height and weight of the person in the photographs relative to an average person. Task instruction in this perceptual context condition was carefully framed to avoid references to social scenarios in order to reduce the activation of socially motivated processing strategies. This allowed direct comparison of memory performance on face-context association between social and non-social encoding prospective. This comparison was chosen to shed light on examining whether the cultural effects in memory for socially meaningful contexts could be equivalently extended to perceptual contexts. It will help to address whether social salience of the encoding context is a critical factor in cultural differences in context memory.

Note that Yang et al. (2013) used only two social context cues, therefore each of the context cue was paired with the same neutral cue across blocks ("typicality in daily life" was paired with "independent" in one block and with "relational" in the other block). However, the present study contained two separate context cues in each context conditions ("friendly" vs. "success" in social context, "height" vs. "weight" in perceptual context). For the purpose of simplicity, the two cues that belonged to the same contextual category were grouped into the same block. This manipulation also reduced the number 
of trials required for the encoding and recognition, which minimized the detrimental influence of fatigue or memory interference on memory performance.

As described earlier (e.g., Yang et al, 2013), both the elaborative encoding instruction and social salience might drive cultural difference in item-context association. Therefore, it was hypothesized that (1) the East Asian participants would perform better on the item-context association memory task than Caucasian participants; and (2) the cultural difference would be greater in the social context condition than in the perceptual context condition. Alternatively, if group differences in memory is only demonstrated in the social condition but not in the perceptual condition, it would mean that the socially salient context is the main cause of cultural differences in the previous studies; if similar culture effects are found across context conditions, it would indicate that the rating instruction might be the main driving factor for the cultural differences. Lastly, if the two culture groups performed equally well across the two context conditions, it would mean that face-context processing was a universal mechanism across culture groups.

The second manipulation examined the potential other race effect on participants' face processing and memory performance. For this purpose, equal numbers of Caucasian and East Asian faces were used to enable participants study both same-race and other-race faces during the experiment. Data analyses were carried out to examine whether the race of presented stimuli influenced participants' memory for target stimuli and the associated context cues. Based on previous literature (e.g., Meissner \& Brigham, 2001), participants from both cultures were expected to show better recognition memory for same-race faces than for other-race faces. With regards to the memory for associated contexts, it is hypothesized that Canadian participants would demonstrate greater 
other-race effect than the Chinese participants, since the culturally diverse processing styles for same vs. other race faces (e.g., Tanaka et al., 2004) may influence the ability to associate face stimuli to its context. It is also possible that the two groups show similar levels of other race effect, suggesting the face-context association processing is operated by mechanisms unaffected by their culturally-influenced processing styles.

In summary, this study aimed to examine the potential cultural differences between East Asian and Western individuals in memory for face stimuli and their associated encoding context cues. In particular, it addressed the following three research questions: (1) Do the two cultures differ in memory for face stimuli and the associated encoding context? (2) Are the cultural differences equivalent across different types of contextual conditions (social vs. perceptual contexts)? And (3) Do the two cultures differ in the other race effect in memory for faces and the associated contexts? 


\section{Method}

\section{Participants}

Thirty-six Caucasian Canadian young adults (27 females; age range $=17-30$ years, $M=21.00, S D=3.94$ ) and Thirty-five Chinese young adults (21 females; age range $=19-27$ years, $M=22.06, S D=2.33$ ) were recruited for this study. The Chi-square test showed that the two culture groups did not differ in gender distribution, $\chi^{2}$ $(1, N=71)=1.82, p=.211$. All the Canadian participants were English-speaking Caucasians with Western European descents, and they were recruited from two sources: (a) undergraduate participant pool via the SONA sign-up system of the Psychology Department at Ryerson University, who received 1\% course credit in their Introductory Psychology courses; or (b) participant recruitment posters distributed on Ryerson University Campus, who received 10 dollars as the compensation for their participation in this typically 1-hour session. All the Chinese participants were native Chinese speakers recruited from the local communities in Beijing and were compensated 30 Yuan for their participation. Canadian participants were tested at the Cognitive Aging Laboratory at Ryerson University whereas the Chinese participants were tested at the Institute of Psychology, Chinese Academy of Sciences in Beijing.

Participant exclusion criteria were applied to participants in both culture groups, and eligible participants (1) were within an age range of 18 to 30 years; (2) had normal or corrected to normal vision; and (3) had never suffered from any psychological or neurological conditions that might impair cognitive abilities. Data from seven Canadian participants were replaced due to incorrect counterbalancing of recognition stimuli. Another four Canadian participants were replaced due to reported head-injury or 
attentional deficits. Data from one Chinese participant was excluded in the final analyses due to high false alarm rates in recognition tasks (see results section for details).

To assess and possibly control for potential cultural differences in social, emotional and cognitive characteristics, a battery of pencil-and-paper questionnaires and culturally appropriate cognitive measures was administered to participants in both groups in addition to the general demographic information sheet. The battery included (1) the Positive and Negative Affect Scale (PANAS; Watson, Clark, \& Tellegen, 1988) that measures the participant's positive and negative affect at the time of experiment; (2) the Self-Construal Scale (SCS; Singelis, 1994), which yields separate scores for independent and interdependent self-construal; (3) a short-version Social Desirability Scale (SDS, Tao, Guoying \& Brody, 2009) that assesses how likely the participant would report favourable responses on self-reports; (4) the Center for Epidemiological Studies Depression Scale (CES-D; Radloff, 1977) that assesses potential depressive symptomatology during the week prior to the experiment; (5) the English and Chinese equivalent vocabulary subset of the third edition of Wechsler's Adult Intelligence Scale ( WAIS -III vocab; Wechsler, 1997) as an indicator of verbal intelligence; (6) a paper version of Pattern Comparison task (Salthouse \& Babcock, 1991) as a measure of perceptual processing speed; (7) a short version of Morningness - Eveningness Questionnaire (MEQ; Horne \& Ostberg, 1976) that measures participant's optimal time of day preference; and (8) a computerized visual-spatial working memory task (VSWM, adopted from Rowe, Hasher, \& Turcotte, 2009) as a measure of non-verbal working memory. All the above questionnaires and tasks either have a comparable Chinese version or were translated into Chinese and back-translated by two bilingual researchers. 
Table 1

Sample Characteristics

\begin{tabular}{|c|c|c|c|c|c|c|}
\hline \multirow[b]{2}{*}{ Measure } & \multicolumn{2}{|c|}{$\begin{array}{c}\text { Canadian } \\
(\mathrm{n}=36,27 \text { females })\end{array}$} & \multicolumn{2}{|c|}{$\begin{array}{c}\text { Chinese } \\
(\mathrm{n}=35,21 \text { females })\end{array}$} & \multicolumn{2}{|c|}{$\frac{\text { Significance }}{\text { (two-tailed) }}$} \\
\hline & Mean & $(S D)$ & Mean & $(S D)$ & $t$ & $p$ \\
\hline \multicolumn{7}{|c|}{ Demographic information } \\
\hline Age (years) & 21.00 & $(3.94)$ & 22.06 & $(2.33)$ & $-1.381 *$ & .173 \\
\hline Education (years) & 14.04 & $(2.64)$ & 15.97 & $(1.82)$ & -3.573 & $<.001$ \\
\hline Health rating $^{\text {a }}$ & 8.15 & $(1.03)$ & 7.86 & $(.69)$ & $1.419^{*}$ & .161 \\
\hline Short MEQ ${ }^{b}$ & 13.38 & $(3.89)$ & 14.37 & $(2.63)$ & $-1.261 *$ & .212 \\
\hline \multicolumn{6}{|c|}{ Emotional and social characteristics } & PANAS $^{\mathrm{c}}$ \\
\hline - positive affect & 26.81 & $(5.97)$ & 30.77 & $(5.68)$ & -2.865 & .006 \\
\hline $\begin{array}{l}\text { - negative affect } \\
\text { SCS }^{d}\end{array}$ & 14.28 & $(5.70)$ & 14.31 & $(4.17)$ & -.031 & .976 \\
\hline - independence & 4.84 & $(.56)$ & 4.14 & $(.73)$ & 4.542 & $<.001$ \\
\hline - interdependence & 4.72 & $(.68)$ & 4.89 & $(.60)$ & -1.145 & .256 \\
\hline $\operatorname{SDS}^{\mathrm{e}}$ & 5.97 & $(3.18)$ & 7.23 & $(2.03)$ & $-1.988 *$ & .051 \\
\hline CES-D ${ }^{f}$ & 16.36 & $(6.36)$ & 13.63 & $(5.97)$ & 1.866 & .066 \\
\hline \multicolumn{7}{|l|}{ Cognitive measures } \\
\hline VSWM accuracy ${ }^{\mathrm{g}}$ & .544 & .180 & .633 & .198 & -1.99 & .051 \\
\hline WAIS - III vocab ${ }^{\mathrm{h}}$ & $57.14^{\mathrm{j}}$ & $(12.10)$ & 62.86 & $(6.87)$ & -2.430 & .018 \\
\hline Pattern comparison ${ }^{\mathrm{i}}$ & 39.69 & $(6.35)$ & 52.14 & $(8.87)$ & $-6.787 *$ & $<.001$ \\
\hline
\end{tabular}

Note. Detailed descriptions of the measures were provided in the methods section.

${ }^{a}$ Health rating were self-reported on a scale of 1 (poor) to 10(excellent).

${ }^{\mathrm{b}}$ Short MEQ have a maximal range of 5.0 - 24.0.

${ }^{\mathrm{c}}$ PANAS scores have a maximal range of $10-50$.

${ }^{\mathrm{d}}$ SCS scores have a maximal range of $1.0-7.0$.

${ }^{\mathrm{e}}$ SDS have a maximal range of $0-14$.

${ }^{\mathrm{f}} \mathrm{CES}-\mathrm{D}$ have a maximal range of $0-60$.

g VSWM accuracy is the proportional correct responses in the 4-7 item trials.

${ }^{\mathrm{h}}$ WAIS - III vocab have a maximal range of $0-80$.

${ }^{\mathrm{i}}$ Pattern comparison has the maximal range of $0-96$.

${ }^{\mathrm{j}} \mathrm{n}=35$, one Canadian participant was excluded due to the poor performance (i.e., 3SD below group mean) as a result of misunderstanding of task instruction in WAIS-III.

* Independent sample $t$-test with equal variances not assumed.

Group differences were found in several measures (see Table 1). Compared to their Canadian counterparts, Chinese participants had more years of education, $t(69)=$ 
$3.57, p<.001, \eta^{2}=.16$; reported higher levels of positive affect, $t(69)=2.87, p<.01, \eta^{2}$ $=.11$; had lower scores on the SCS-independence scale, $t(69)=-4.54, p=.006, \eta^{2}=.23$; as well as showing higher performance on the WAIS-III vocabulary task, $t(69)=2.43, p$ $=.018, \eta^{2}=.08$; and the Pattern Comparison task, $t\left(61.49^{1}\right)=6.79, p<.001, \eta^{2}=.43$. All the other demographic measures were matched between the two culture groups. Despite groups differences in these measures, none of these measures were found to correlate with memory performance, except for the levels of positive affect, which was negatively correlated with context memory scores in the social context block, $r(71)=-.320, p$ $=.007$. However, analyses of covariance (ANCOVA) that controlled for this variable didn't change the statistical significance of main results in context or culture effect. This suggests that these demographic differences between the two samples did not account for the effects observed in this study.

\section{Stimuli and Apparatus}

One hundred and six coloured photographs of human faces with neutral expressions were used in this study. Half of them were East Asian faces from a previous cross-cultural face learning study (Yang, Chen, Ng, \& Fu, 2013) and the face database from Visual Cognition Laboratory at University of Hong Kong (Hayward, 2012); the other half were Caucasian faces selected from the Center for Vital Longevity at University of Taxas at Dallas face database (Minear \& Park, 2004). Both the East Asian and Caucasian face sets contained equal number of male and female faces, as well as equal number of younger (ages 18 - 30) and older (ages 60 - 80) faces. Each of the photographs showed a front-facing pose against a white background, with their face

\footnotetext{
1 Adjusted degrees of freedom was used because independent sample $t$-test on this task violated the equal variance assumption by Levene's test, $p=.015$.
} 
taking approximately $60 \%$ of the space in the center. Ten of the photographs were used in practice trials, and the remaining 96 were used in the actual encoding and recognition trials. The 96 photographs for the actual experiment were sorted into six sets of 16 faces, with age, gender and ethnicity of the faces being evenly distributed in each set. These sets were then counterbalanced across the two context condition blocks and each list was equally likely to serve as an old list for each of the two context conditions or the non-studied new list in the recognition task. Since the other race effect was examined in the study, participants from both culture groups viewed the identical set of stimuli, half are of the same race and half are of the other race.

All the computer tasks were created and run in E-Prime 2.0 software (Psychology Software Tools, Inc., Pittsburgh, PA). All the task instructions and stimuli were presented centrally against a white background. The tasks were presented with 15-inch laptop computers at both testing sites. All the tasks were adjusted to $800 \times 600$ resolution on the testing computer monitor. The operating system on Canadian testing laptop was Windows 7 while the Chinese testing laptop was running Windows XP. At both testing sites, participants were comfortably seated in a well-lit testing room with a viewing distance of approximately $50 \mathrm{~cm}$ (19.7 inch) between the participant and laptop monitor.

\section{Procedure}

After being greeted, the participant was led to the experiment room and signed the consent form. Then the participant was seated in front of the testing computer and completed two blocks of context memory tasks: a social context memory block and a perceptual context memory block. Each block took approximately 10 minutes to 
complete excluding practice time. The order of the two blocks was counterbalanced across participants.

Both of the blocks contained an encoding phase and a recognition phase. The study phase of each block consisted of 32 trials. At the beginning of each study trial, a fixation cross appeared at the center of the screen for $1000 \mathrm{~ms}$, prompting the participants to pay attention to the upcoming trial. Then a photograph of a human face appeared, along with a cue word presented above it. In the social context block, half of the trials were paired with the cue word “FRIENDLY” (“友好” for Chinese) and the other half were paired with “SUCCESS” (“成功” for Chinese). In the perceptual context block, the cue word were “HEIGHT” (“身高” for Chinese) for half of the trials and “WEIGHT” (“体重” for Chinese) for the other half. The encoding trials were pseudo-randomized so that no more than three trials with the same cue were presented consecutively in any block or counterbalance condition.

Participants were instructed to carefully examine the photographs and evaluate the faces based on the cue word (See Table 1 for detailed instruction for each cue) by pressing the corresponding number keys on the keyboard. The photograph remained on the screen for $5000 \mathrm{~ms}$ and was then replaced by a blank screen for $1000 \mathrm{~ms}$ before proceeding to the next trial. Participants were told that the photograph would remain on the screen for $5000 \mathrm{~ms}$, and were encouraged to take their time to provide the response within that time window. The test phase started immediately after the encoding phase. 
Table 2

Encoding Task Instructions

\begin{tabular}{|c|c|c|}
\hline Block & Cue A & Cue B \\
\hline & FRIENDLY & SUCCESS \\
\hline $\begin{array}{c}\text { Social } \\
\text { Context }\end{array}$ & $\begin{array}{l}\text { "Imagine this person standing in } \\
\text { front of you. How friendly do you } \\
\text { think this person is relative to an } \\
\text { average person? Please use a scale of } \\
1 \text { to } 5 \text {, with } 1 \text { being not friendly at all } \\
\text { and } 5 \text { being very friendly." }\end{array}$ & $\begin{array}{l}\text { "Imagine this person standing in } \\
\text { front of you. How successful do you } \\
\text { think this person is relative to an } \\
\text { average person? Please use a scale of } \\
1 \text { to } 5 \text {, with } 1 \text { being not successful at } \\
\text { all and } 5 \text { being very successful." }\end{array}$ \\
\hline & HEIGHT & WEIGHT \\
\hline $\begin{array}{c}\text { Perceptual } \\
\text { Context }\end{array}$ & $\begin{array}{l}\text { "Imagine this person standing in } \\
\text { front of you. How tall do you think } \\
\text { this person is relative to an average } \\
\text { person? Please use a scale of } 1 \text { to } 5,1 \\
\text { being very short and } 5 \text { being very } \\
\text { tall." }\end{array}$ & $\begin{array}{l}\text { "Imagine this person standing in } \\
\text { front of you. How heavy do you } \\
\text { think this person is relative to an } \\
\text { average person? Please use a scale of } \\
1 \text { to } 5,1 \text { being very light and } 5 \text { being } \\
\text { very heavy." }\end{array}$ \\
\hline
\end{tabular}

There were 48 trials in the test phase, including 32 old photographs from the study phase (16 paired with each cue word) and 16 new photographs which were never displayed in the experiment. Each test trial started with a $1000 \mathrm{~ms}$ fixation cross, followed by the an old or new face presented for $5000 \mathrm{~ms}$. Participant were instructed to indicate, via designated key press responses, whether each stimulus was (a) studied during encoding phase (old) and were rated in context A (i.e., under the cue word "FRIENDLY" in the social context block or "HEIGHT" in perceptual context block); (b) old and were rated in context B (i.e., under the cue word "SUCCESS" in the social context block or "WEIGHT" in perceptual context block); or (c) not seen during the study phase (new). The recognition trials terminated when a response was made or 5000 
$\mathrm{ms}$ has passed. Then a blank screen appeared for $1000 \mathrm{~ms}$ before proceeding to the next trial. The corresponding keys for the recognition responses were switched and counterbalanced between participants. Half of the participants were instructed to respond by pressing the keys " $\mathrm{z}$ " (representing "FRIENDLY" or "HEIGHT"), "x" (representing "SUCCESS" or "WEIGHT") and "/" (representing "NEW") using their left middle and index fingers and the right index finger respectively; whereas for the other half of the participants, the keys “/” (representing "FRIENDLY” or "HEIGHT”), "." (representing "SUCCESS" or "WEIGHT") and "z" (representing "NEW") were assigned to the right middle and index fingers and the left index finger, respectively. Coloured stickers with corresponding cue words were taped on the keyboard to demonstrate the respective key-response relationships. Participants were instructed to rest their fingers on the three response keys throughout the recognition phase and respond as quickly and accurately as possible.

Participants were trained on four practice trials during the encoding phase (two trials with each cue) and 6 practices trials on recognition phase before performing the actual task. They were given the opportunity to repeat the practice trials several times if they were uncertain about task instructions. Figure 1 displays an example of the study and recognition trial paradigm. 


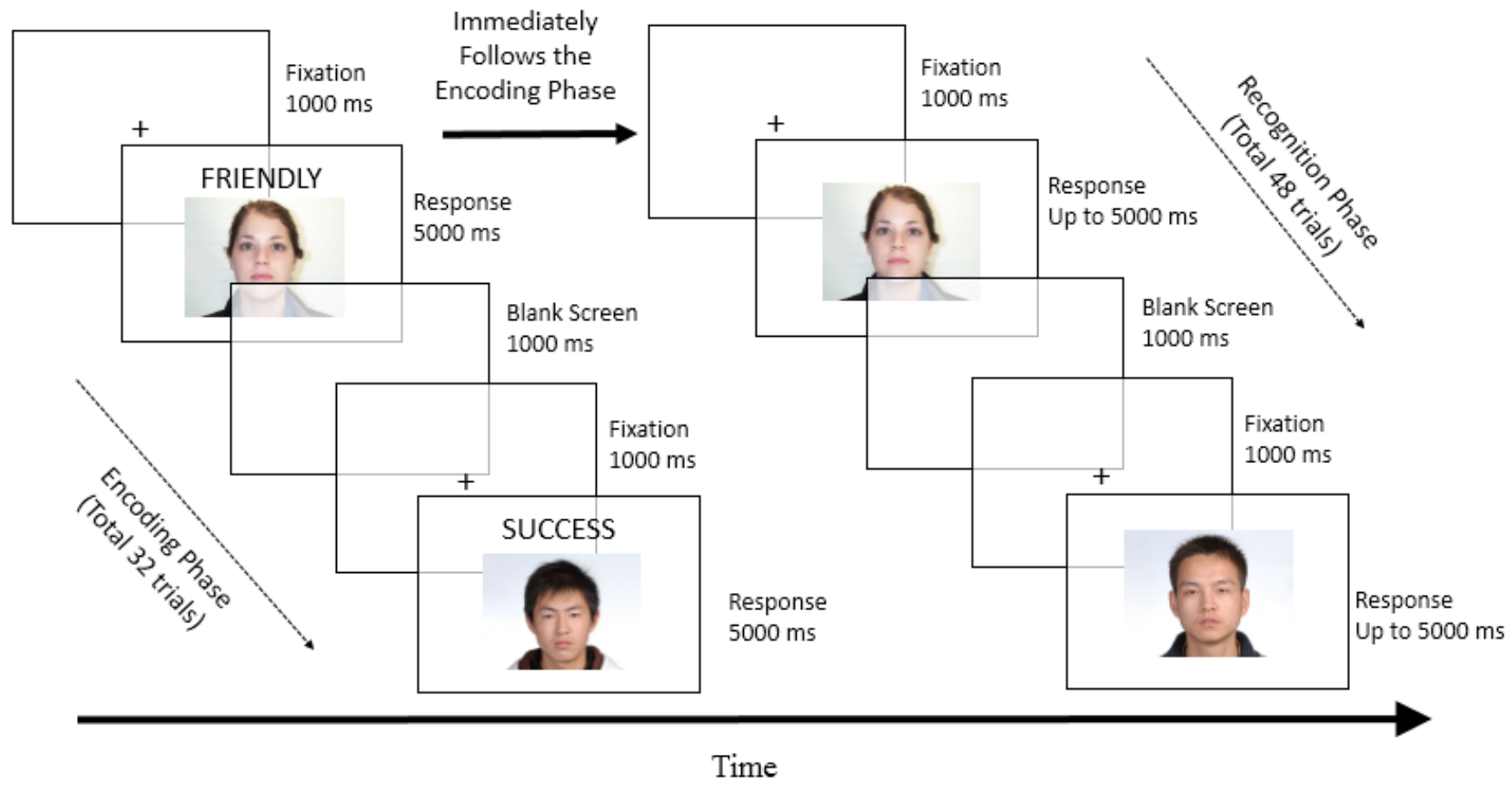

Figure 1. Example of the experimental paradigm in the social context block. During the encoding phase, each trial will consist of a face and a cue word presented above it. The cue words are either "FRIENDLY" or "SUCCESS" in the social block; or either "HEIGHT" or "WEIGHT" in the perceptual block (corresponding Chinese words were used for Chinese participants in Beijing). Participants were instructed to respond using a scale from 1 to 5 based on the cue. During the recognition phase, participants viewed face stimuli and indicate whether each face was a new face or an old one rated in one of the two specific contexts. Both the keyboard input and the response times (RTs) were recorded by the e-prime program.

A filler task, the computerized non-verbal visual spatial working memory task (VSWM, modified from Rowe et al., 2009), was administered between the two blocks to reduce interference between the two context memory blocks. In this task, participants were instructed to remember the order of squares that were lit up during each trial, and later reproduce the sequence of presentation with mouse clicks. All the participants were given 6 practice trials before starting the actual task. The length of sequence varied 
between 4 to 7 squares with increasing difficulty. Performance on this task could be used as an indicator of one's non-verbal visual spatial working memory capacity.

Upon completion of the two context blocks and the filler task, participants were instructed to complete a photo difficulty rating questionnaire (see Appendix IV). This questionnaire required the participants to report the overall difficulty they experienced with the encoding tasks, based on a Likert-type scale of 1 to 7 , with higher number representing greater difficulty experienced during the tasks. The questionnaire also probed the participants to report (1) if they had any difficulty with a particular type of stimuli and/or cue word, as well as (2) if they utilized any self-generated strategies in addition to the task instruction to aid the rating/encoding of the stimuli. Then the participants completed the battery of emotional, social and cognitive measures in the presented order: the PANAS, SCS, SDS, CES-D, WAIS-III vocab, Pattern Comparison, MEQ, and a demographic information sheet (information collected including participant's age, gender, ethnicity, native language, handedness, level of education and physical health). At the end, participants were fully debriefed and compensated. 


\section{Results}

\section{Statistical Analyses}

All statistical analyses were conducted using SPSS 20.0, with a significance level set at .05 and marginal significance level set at .10 unless specified otherwise. A set of 2 by 2 mixed model analyses of variance (ANOVAs) were conducted on encoding and recognition performance, with culture (Canadian vs. Chinese) as a between-subject factor and context block (perceptual vs. social) as a within-subject factor.

Trials with RTs of 0 ms were treated as timeout while trials with RTs below 50 ms were treated as immature key press error (instead of deliberate response made by the participant). To avoid skewing the RTs data, these trials were excluded in the final analyses. Overall, $1 \%$ of encoding trials and $.4 \%$ recognition trials were excluded in this experiment, comparable to the previous study (Yang et al., 2013).

In addition, to check for potential outliers in memory performance, the mean false alarm rates (percentage of new trials incorrectly identified as old) across the two context memory blocks were calculated for each participants. High false alarms might indicate misunderstanding of instruction or failure to consistently follow the key designation in their responses. Individuals who scored 3 standard deviations above the mean in the respective culture group were regarded as outliers and excluded from the final analyses. As described previously in the methods section, the data from one Chinese participant was excluded due to this false alarm check. None of the Canadian met the exclusion criterion. 


\section{Encoding Performance}

Stimuli Rating. Group means in rating scores and RTs during encoding are displayed in Table 3. The analysis on rating scores showed no main effect of culture, $p$ $=.808$. However, there was a significant main effect of context, with stimuli in the perceptual block $(M=3.10, S D=.22)$ rated higher than those in the social block $(M=$ $3.01, S D=.36), F(1,69)=4.545, p=.037, \eta_{p}{ }^{2}=.062$. This effect was qualified by a significant context by culture group interaction, $F(1,69)=10.137, p=.002, \eta_{p}^{2}=.128$. Follow up analyses showed that the context effect on rating score was only significant for the Chinese group, $F(1,34)=17.86, p<.001, \eta_{p}{ }^{2}=.344$, but not for the Canadian group, $p=.501$. This difference in perceptual rating response is likely due to the different body build norms between East Asian and Western cultures, rather than reflecting cultural differences in encoding processes.

Encoding RTs. The mixed model ANOVA on encoding RTs showed no main effects of culture or context, $p s>.34$, nor any culture by context interaction, $p=.63$. Table 3

Group Means in Encoding RTs and Rating Scores

\begin{tabular}{lcccc}
\hline & \multicolumn{2}{c}{ Canadian $(\mathrm{n}=36)$} & & \multicolumn{2}{c}{ Chinese $(\mathrm{n}=35)$} \\
& Mean & $(S D)$ & Mean & $(S D)$ \\
\hline Rating Score (1 - 5) & 3.04 & $(0.19)$ & 3.17 & $(0.24)$ \\
Perceptual Block & 3.09 & $(0.38)$ & 2.93 & $(0.32)$ \\
Social Block & & & & \\
Encoding RTs (in ms) & 2513 & $(359)$ & 2613 & $(430)$ \\
Perceptual Block & 2545 & $(369)$ & 2611 & $(440)$ \\
Social Block & & & & \\
\hline
\end{tabular}




\section{Recognition Performance}

For each context block, item memory accuracy was calculated by subtracting the percentage of new items incorrectly identified as old (false alarms) from the percentage of old items correctly identified as old (hits). The context memory accuracy, on the other hand, was calculated by dividing the total percentage of old items attributed to the correct sources by the total number of old items correctly identified as old (hits). The calculation of item and context memory was modeled after Rahhal, May \& Hasher (2002). Table 4 displays the group means of hit rates, false alarm rates, item memory and context memory scores during the recognition phase.

Table 4

Group Means in Memory Scores (in percentage) During Recognition

\begin{tabular}{lcccc}
\hline & \multicolumn{2}{c}{ Canadian $(\mathrm{n}=36)$} & \multicolumn{2}{c}{ Chinese $(\mathrm{n}=35)$} \\
\cline { 2 - 3 } & Mean & $(S D)$ & Mean & $(S D)$ \\
\hline Perceptual Block & 80.85 & $(10.42)$ & 77.83 & $(13.84)$ \\
False alarms & 14.11 & $(13.05)$ & 14.84 & $(12.23)$ \\
Item recognition & 66.74 & $(16.29)$ & 62.99 & $(14.97)$ \\
Context attribution & 65.41 & $(12.21)$ & 62.63 & $(11.82)$ \\
Social Block & & & & \\
Hits & 85.92 & $(10.22)$ & 83.88 & $(10.48)$ \\
False alarms & 9.23 & $(8.12)$ & 13.11 & $(11.68)$ \\
Item recognition & 76.69 & $(12.59)$ & 70.77 & $(12.84)$ \\
Context attribution & 74.57 & $(11.28)$ & 72.24 & $(12.50)$ \\
\hline
\end{tabular}

Item memory. As described above, item memory scores were corrected by subtracting the hit rates by the false alarm rates. ANOVA revealed a main effect of 
context, $F(1,69)=34.198, p<.001, \eta_{p}{ }^{2}=.331$, with significantly higher item memory in the social block $(M=73.77, S D=12.97)$ than in the perceptual block $(M=64.89, S D=$ 12.97). However, neither the main effect of culture nor the culture by context interaction was significant, both $p s>.11$.

Context memory. The ANOVA showed a main effect of context, $F(1,69)=$ $28.733, p<.001, \eta_{p}{ }^{2}=.294$, with significantly higher context attribution accuracy in the social $(M=73.42, S D=11.87)$ than in the perceptual block $(M=64.04, S D=12.02)$. There was no significant main effect of culture or culture by context interaction, both $p s>.25$.

Recognition RTs. Figure 2 shows the mean recognition RTs for each culture group. ANOVA revealed a main effect of culture, $F(1,69)=8.253, p=.005, \eta_{p}{ }^{2}=.107$. Chinese participants responded significantly slower $(M=2279.14, S D=464.45)$ than did Canadian participants $(M=1980.13, S D=411.22)$. There was also a significant main effect of context, $F(1,69)=8.046, p=.006, \eta_{p}^{2}=.104$, with slower response time during perceptual block $(M=2188.79, S D=556.78)$ than in the social block $(M=2066.27, S D$ $=434.12$ ). These effects were qualified by the significant context by culture interaction, $F$ $(1,69)=7.74, p=.007, \eta_{p}^{2}=.101$. Follow up analyses revealed that Chinese participants were significantly slower in the perceptual block $(M=2402.06, S D=531.66)$ than in the social block $(M=2156.20, S D=482.47), F(1,34)=12.607, p<.001, \eta_{p}^{2}=.270$; while no context effect was found in the Canadian participants, $p=.97$.

To further examine whether the slower responses in Chinese reflects a general cultural bias in response speed or an effect specific to context attributions, a follow-up ANOVA was conducted on the recognition RTs for trials with correct rejection (new item 
correctly identified as "NEW"). This time, neither the main effect of culture nor the culture by context interaction was significant, both $p s>.56$. This suggests that the slower recognition RTs in Chinese participants, particularly for the perceptual context condition, were specifically related to trials for which they had to attribute the old face stimuli to a specific context cue, but not the generally slower response time in recognition tasks. Therefore, the results suggest that Chinese participants possibly experienced greater difficulty, as manifested in taking longer time to provide responses, to retrieve face-context association information than Canadian participants in the context memory task.

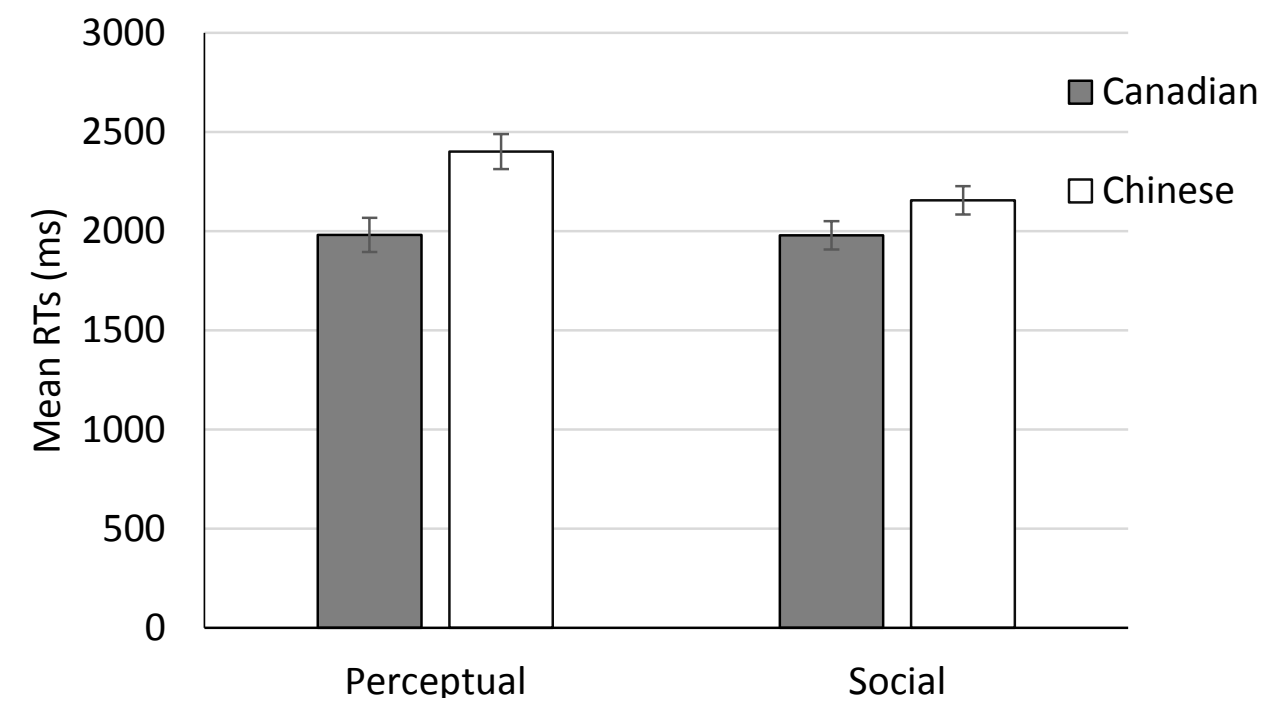

Figure 2. Group means of recognition RTs in perceptual and social context blocks

\section{The Other Race Effect on Memory Performance}

To investigate the potential cultural differences in the other race effect in memory performance, additional analyses were conducted with trials separated into two conditions: same-race and the other-race condition. Trials in which Chinese participants were shown Asian face stimuli and Canadian participants were shown Caucasian face 
stimuli was marked as "same-race" condition, all the other trials were marked as "other-race condition". Then a 2 (race: same-race vs. other-race) by 2 (culture: Canadian vs. Chinese) by 2 (context: social vs. perceptual block) mixed model ANOVA was conducted on provided rating as well as item and context memory performance.

Item memory. Group means of item memory performance were illustrated in Figure 3. The analysis revealed a main effect of race, $F(1,69)=18.457, p<.001, \eta_{p}{ }^{2}$ $=.211$, with higher accuracy in the same-race condition $(M=73.50, S D=13.05)$ than in the other-race condition $(M=64.87, S D=17.49)$. None of the other main effects or interactions were significant, all $p s>.15$.

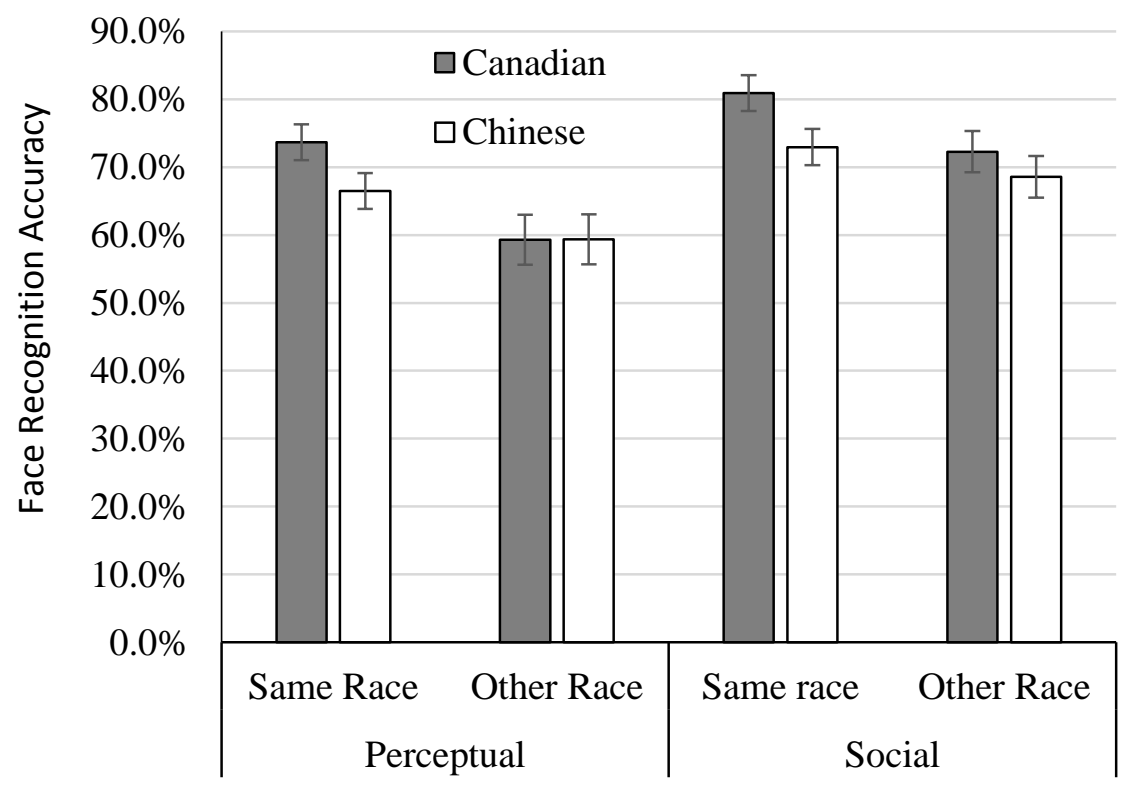

Figure 3. Group mean item memory scores across context blocks and races

Context memory. The results showed a marginally significant main effect of race, $F(1,69)=3.814, p=.055, \eta_{p}{ }^{2}=.052$, with higher context attribution accuracy in the same-race condition $(M=70.14, S D=11.87)$ than in the other-race condition $(M=67.10$, $S D=11.31$ ). This effect was qualified by a marginally significant race by culture 
interaction, $F(1,69)=3.369, p=.071, \eta_{p}^{2}=.047$. To verify the hypothesis on potential cultural difference in the influence of other race effect, follow-up analyses were conducted to examine other-race effect separately for each culture group. Results showed a significant same-race advantage effect only in Canadians, $F(1,69)=8.139, p=.007$, $\eta_{p}{ }^{2}=.189$, but not in Chinese, $\mathrm{p}=.938$. This interaction was displayed in Figure 4 .

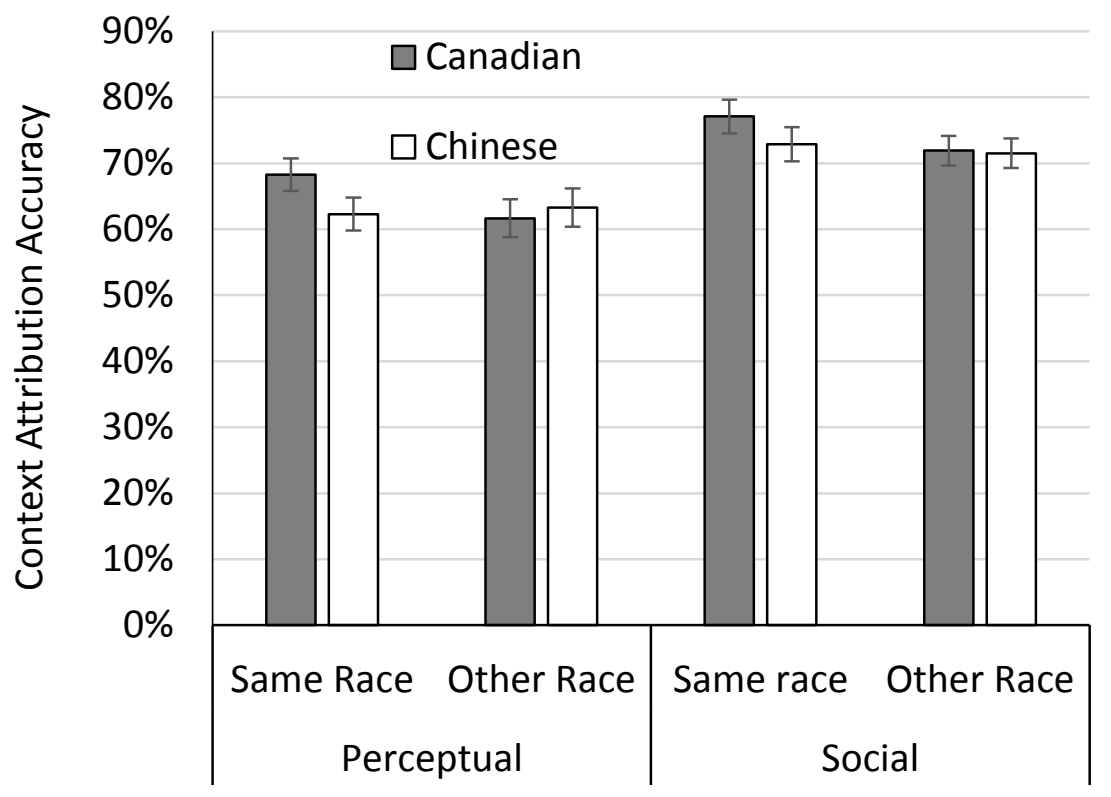

Figure 4. Group mean context memory scores across context blocks and races

In summary, these results replicated previous findings of the other race effect in face recognition literature (Meissner \& Brigham, 2001). In item memory, both Canadian and Chinese participants were more accurate at recognizing faces of the same race than those of another race. Findings of the current study also provide potentially novel insight that the Canadians but not Chinese demonstrated the other race effect in memory for the face-context associations. Since this analysis is based on marginal interaction, implications need to be taken with caution. 


\section{Task Difficulty and Strategy Use}

Self-reported difficulty. To check whether the perceived level of difficulty at encoding was different across the two culture groups, the mean rating on the photo difficulty rating questionnaire was compared. Result showed no cultural difference between Canadian $(M=4.00, S D=1.29)$ and Chinese groups $(M=4.03, S D=1.04), t$ $(69)=-.103, p=.918$. This suggests that two cultural groups did not differ in perceived task difficulty level at encoding.

Strategy use. The photo difficulty rating questionnaire also probed the potential use of self-generated encoding strategies in addition to the task instruction. A few participants in the Canadian sample reported using self-generated strategies during the encoding task. In particular, one Canadian participant reported judging the perceived height and weight based on the location of the face inside the frame. Five Canadian participants reported picturing someone they knew in life that resembled the presented stimuli as reference, and estimated the friendliness based on their experience with the reference person. None of the Chinese participants reported using any self-generated strategies on the questionnaire. The main results on memory performance remained the same when data from the participants who reported additional strategy use was removed in the analyses. 


\section{Discussion}

This study aimed to investigate three primary research questions: (1) Do cultural differences exist between East Asian and Western young adults in their memory for face stimuli and the associated encoding context cues when the association is established with holistic encoding processes? (2) Are the cultural differences, if any, equivalent across social and perceptual contexts? And (3) does the other race effect influence the two cultures differentially for item and context memory for face stimuli? To address these questions, the present study manipulated the experimental instructions to separately promote perceptual-focused and social-focused encoding contexts for face processing during the encoding phase. Then the memory for the face stimuli and associated contexts were assessed in the recognition task in which Canadian and Chinese participants identified whether a testing face stimulus was a new face or an old one studied in reference to a specific context cue.

\section{The Unique Mechanisms Underlying Face Processing}

This study did not replicate the East-Asian advantage in object-context association memory observed in Yang et al. (2013). Overall, this experiment did not detect any cultural difference in item or context memory performance for face stimuli between the Chinese and Canadian participants. The only cultural difference was found in the recognition RTs. Chinese participants showed slower response time than Canadian participants on context attribution trials, particularly in the perceptual context block. This result indirectly suggests that Chinese participants may have experienced greater difficulty attributing a studied face to its corresponding context cue (particularly in the perceptual contexts) than Canadian participants. The discrepant results between the 
current study and Yang et al. (2013) might reflect underlying differences in processing and memory mechanisms between face-context association and object-context association.

As reviewed previously, face processing and recognition demand higher levels of holistic processing of the stimuli than most object processing tasks (Richler et al., 2011; Wang et al., 2012). At the same time, object processing literature has shown that East Asian participants habitually engaged in more holistically oriented information processing styles. However, results in the current study did not show any East Asian advantage in a task that apparently favors their culturally preferred processing styles.

It is possible that the holistic representation of facial information that East Asian participants formed during encoding could not promote effective face-context association and recognition in this particular task. As mentioned previously, most research on face processing has focused on the recognition of face stimuli itself. Even though the literature suggests that face recognition heavily depends on holistic processing, no research has examined the association of the contextual information to face stimuli. It is possible that the specific task of associating context cues to a human face demanded more feature-specific details than the holistic representation of an object within a context. For instance, when instructed to study an object (such as in Yang et al., 2013), one could holistically incorporate personal experiences and semantic knowledge related to that object in a meaningful way (e.g. "an apple is very useful in my independent life because it keeps me healthy”) without considering the specific perceptual details; whereas this strategy may not be useful or even possible for the encoding of face stimuli since all of 
human faces were structurally similar with slight differences in spatial configuration and facial features.

This speculation is supported by face memory research. Dewhurst, Hay, \& Wickham (2005) examined the relationship between different dimensions of face assessment (familiarity, distinctiveness, attractiveness, typicality, etc.) and the recollective experiences (remember vs. know) in face recognition task. Their found that the distinctiveness of the given faces (e.g., containing distinguishing structural or facial feature) determined how likely they will be recollected as "remembered", whereas all the other factors were related the familiarity-based recognition ("known"). Along the same line, O’Toole and colleagues (1994) demonstrated that the memorability of a particular human face was related to the small, local, and distinctive features, whereas the familiarity aspect of memory was related to the global, configuration-based information in the face. It is possible that a holistic processing style can only promote effective familiarity-based face recognition (e.g., "I think I've seen this before because this person looks familiar") but not the recollection-based memory with the specific episodic details (e.g., "I estimated this person with the cue word "FRIENDLY", because I remember giving a high rating score based on his/her bright, friendly-looking eyes"). In this study where participants were required to choose one cue word over the other, the former recognition mechanisms would not directly aid the participant to make the correct response. This would also explain why the face-context association task did not demonstrate similar cultural difference from previous findings with objects. Some may argue that the failure to detect cultural difference in face-context association memory could be due to the manipulation in the experimental design. Since 
only social context cues were used, Yang et al. (2013) separated the "independent" and "relational" conditions by pairing them to the same third "typicality in daily life" context as reference. However, the present study directly compared the two same-category context cues within the same block. It is possible that the participants utilized similar encoding strategies within a context ("This person looks very attractive, therefore he/she could be both very friendly and very successful in life") which made it harder for them to differentiate between the two contextual cues in the recognition task. As a result, any cultural difference in item-context association memory may be wiped out by this design.

To verify this assumption, a follow-up experiment was conducted using object stimuli (line-drawing pictures, similar to the ones used in Yang et al., 2013) under the same design with two same-category cues included with a context block. Preliminary analyses $(n=24)$ did not support this speculation that grouping same-category context cues within a block would wipe out cultural differences in item-context association. Instead, it replicated the finding in Yang et al. (2013) in showing that Chinese participants were slightly better at associating studied objects with the corresponding context cues in memory than the Canadian participants. It further supports the notion that the East-Asian advantage in object-context association memory observed in previous study cannot be directly generalized to the memory for face-context associations.

It appears that separate mechanisms may be involved in face-context association and object-context association tasks. It is possible that the face-context association demands more processing of featural information and thus reflects more fundamental cognitive mechanisms that are universal across cultures. In contrast, object-context processing may engage more knowledge-based cognitive pragmatics based on everyday 
experience with the meaningfulness/ functions of the objects, which is thus more subject to cultural variations (Baltes, 1993).

\section{Perceptual vs. Social Context in Face Processing}

A robust main effect of context was observed in both item and context memory performance in this study. For both Canadian and Chinese participants, the faces studied in reference to socially meaningful context cues were more accurately recognized than those studied in reference to perceptual cues. This is consistent with previous finding that people are particularly proficient in making social judgements in face processing tasks (e.g., Todorov et al., 2013). It is possible that the socially salient condition promoted deeper levels of processing and better encoding strategies to form effective association between the face stimuli and the contextual details, resulting in overall better memory performance for the social context condition than the perceptual context condition.

In is worth noting that no culture by context condition interaction was found in memory performance. Thus, it appears that the improved item and context memory in social condition over the perceptual condition was equivalently demonstrated in the two culture groups. This finding suggests that, at least for face stimuli, the social salience of contextual information in item recognition is not more beneficial in one culture over the other.

\section{The Other Race Effect}

The results demonstrated a robust other race effect in face recognition across the two culture groups: for both Canadian and Chinese participants, face stimuli that belonged to the same racial group of the participants were better recognized compared to faces that belonged to another race. This finding replicated previous research on the other 
race effect in the face processing literature (e.g., Meissner, 2001). Since no culture by race interaction was observed, it suggests that the other race effect influenced participants' item memory for face stimuli similarly across the two cultures.

As demonstrated in the results, a marginal culture by race interaction was revealed in context memory performance. Only Canadian, but not Chinese participants, demonstrated the substantial other race effect in their ability to attribute the studied face to the corresponding context cues. This finding replicated the cultural difference in processing styles for same-race and other-race faces observed in Tanaka et al. (2004), in which the authors attributed the cultural difference in processing styles to the greater social exposure experienced by the Asian participants. Unfortunately, this social exposure interpretation cannot apply to the current study. Due to the fact that Toronto is a heavily multi-cultural city with more than $10 \%$ of residents of East Asian background (Statistics Canada, 2011), Canadian participants recruited in Toronto are expected to have higher levels of social interaction with East Asian individuals compared to Chinese participants recruited in Beijing who lived in China and have limited opportunity to interact with individuals from the Western culture. If social exposure determines how face stimuli are processed as suggested in Tanaka et al. (2004), then the Canadian participants should show a smaller other race effect in memory performance than their Chinese counterparts. Alternatively, it is possible that Canadian participants were more effective at extracting featural information in Caucasian faces, providing them the opportunity to process these faces more holistically; whereas the Chinese participants may solely rely on the holistic processing style regardless of the race of face stimuli. This theory is supported by the 
eye-tracking evidence that East Asian adults viewed visually homogeneous items holistically, even when the item was not a human face (Kelly et al., 2010).

\section{Limitations and Future Directions}

The current study has a few limitations. First, due to the limited cultural difference observed in memory performance, the present study could only interpret the finding based on previous literature in face recognition research. To gain better understanding of the underlying mechanisms involved in face-context association across cultural groups, future studies should consider comparing behavioral patterns and neural activities exhibited by East Asian and Western participants during the encoding and recognition phase. For instance, eye-tracking technique could be used to examine whether recognition performance would be better when participants make more fixations to specific facial features such as the eyes. In addition, neuroimaging studies could be conducted to compare brain activation patterns during encoding and recognition phase, in order to determine whether the face-context association process is truly universal across culture groups.

Another limitation is that the current design cannot rule out the possibly that the diminished culture difference in item-context association could apply to any other visually homogenous stimuli. One study by Kelly, Miellet and Caldara (2010) compared eye movement patterns between East Asian and Western observers when they were learning and recognizing human faces and other human-face-like objects (sheep faces and computer generated Greebles). They found that this feature vs. configuration disparity between East Asians and Westerners in visual attention was also demonstrated in the viewing of sheep faces and Greebles. Future studies may consider using the current 
paradigm to investigate whether cultural difference will be found in visually similar, but non-human stimuli such as animal faces, or multiple versions of a single category of common object (e.g., different types of dogs).

Lastly, although sample characteristics such as affective states, visual spatial working memory, verbal intelligence and self-construal were measured and inspected as potential source of confound, none of the factors seemed to relate to the memory performance in the current study. At the same time, the cultural differences in the independence score on the Self-Construal-Scale were not predictive of their performance on the memory task. Future cross-cultural studies should consider utilizing measures that are more closely related to the holistic/analytic processing styles between cultures, such as the Analytic-Holism Scale (Choi et al., 2007), and determine whether they were indeed underlying cultural differences in item-context association for face stimuli.

\section{Conclusion and Implications}

In summary, the current study examined the potential cultural differences between Chinese and Canadian younger adults in their ability to form effective association between human face stimuli and the corresponding actively processed context cues. Both social and perceptual contexts were employed to examine whether the cultural effect was exclusive to social scenarios. At the same time, the potential influence of the other race effect in face processing was also examined.

Results showed that both face recognition memory and the face-context association were more accurate in the socially meaningful condition than in the perceptual condition. However, no cultural difference in memory performance was observed, nor was it influenced by the type of encoding context. I argue this lack of 
cultural difference in the current study could be caused by the unique mechanisms underlying face-processing and face-context association. It is possible that the specific face-context association task demands greater processing of perceptual and featural details than the holistic information of the whole face. In addition, the study yielded an interesting cultural difference in the manifestation of the other race effect in context memory. More research is needed to better understand the true nature of face-context association as well as the potential cultural effect involved.

These findings contributed to the scientific understanding of cross-cultural research on item-context association between East Asian and Western cultures. To my knowledge, this is the first study to investigate the ability to associate face stimuli with context cues, not to mention cross-culture comparisons. It offered novel perspectives to both the field of cross-cultural comparison on information processing styles and the research on face recognition mechanisms. The study demonstrated that socially meaningful contextual cues may elicit stronger and more reliable association between a face and its associated context cues, equivalently for both East Asian and Western individuals. Moreover, findings of this study could have significant social impact. In multicultural countries such as Canada, a significant portion of population consists of non-Western immigrants and residents. It is crucial to realize that individuals from different cultural backgrounds may have differential attention and memory for perceived information of a specific face of own or other races. This understanding can inform effective interpretation and policy making in law enforcement, such as interpreting eye-witness report towards a minority suspect. 


\section{Appendix I: Participant Consent Forms (Community Recruitment)}

\section{RYERSON}

UNIVERSITY

\section{CONSENT TO PARTICIPATE IN RESEARCH Cross Cultural Comparison in Memory Binding (Community Recruitment)}

You are being invited to participate in a research study. Please read this Consent Form so that you understand what your participation will involve. Before you consent to participate, please ask any questions necessary to be sure you understand what your participation will involve.

INVESTIGATORS: This research study is being conducted by Lingqian Li, Master student, Department of Psychology, under the supervision of Dr. Lixia Yang, associate professor, Department of Psychology, Ryerson University. Results of this study will contribute to a Master's thesis study of the principal investigator. This study is sponsored by Canadian Institutes of Health Research (CIHR).If you have any questions or concerns about this study, please contact Lingqian Li, at lingqian.li@ psych.ryerson.ca, or 416-979-5000, ext.4987.

PURPOSE OF THE STUDY: This study aims to examine how cultural background could affect people's ability to bind information in memory.

DESCRIPTION OF THE STUDY AND YOUR PARTICIPATION: The study is constituted of one session, which will take approximately one hour to complete. It will take place in the Cognitive Aging Laboratory at the Psychology Research and Training Centre of Ryerson University (105 Bond St., Toronto). Prior to the task, the study procedure will be explained and you will have opportunity to ask questions.

If you volunteer to participate in this study, you will be asked to do the following things during the experiment:

- Computerized tasks: you will be required to view and study some pictures on the computer, then make responses as instructed by the experimenter.

- Paper-and-pencil tests and questionnaires: you will be asked to complete a set of questions that include queries on: demographic information (e.g., age, gender, and native language), education (e.g., the number of years at each educational institute), and personal health (e.g., illness, vision, hearing problems, any previous head injuries, and current medication use); emotion (e.g., describe your feelings at the moment); and vocabulary.

We request all the above information in order to assess what factors have effects on the tasks we ask you to perform today. You may refuse to answer any particular questions or any tasks without any negative consequence. You will be fully debriefed at the end of the session, any questions you may have about this study will also be answered. If you are interested to know the research findings of this experiment, you can choose to notify the experimenter. A copy of the published work can be made available to you upon publication.

EXPERIMENTAL ASPECTS OF THIS STUDY: None of the procedures or questionnaires used in this study is experimental in nature, in the sense that they have all 
been used by other researchers and found to be safe and useful. From a procedural point of view, part of this study is considered "experimental" because by following the procedure described above, the study examines the impact of some variables (called the "independent variables") on other variables (called the "dependent variables"). More information about the variables will be provided at the end of the study.

POTENTIAL RISKS AND DISCOMFORTS: There is minimal to no anticipated risk involved in this study. The tests used in this study are standardized psychological tests and the pictures used can be frequently encountered in newspaper, radio, TV, or movies. Every effort will be taken to ensure that you are as comfortable as possible throughout the session. If any of the pictures or questions makes you feel discomfort, you can take a break, choose not to answer a particular question, or discontinue participating, for any reason during the experiment.

POTENTIAL BENEFITS TO PARTICIPANTS AND/OR TO SOCIETY: The anticipated benefits of participation in this study include: 1) to gain knowledge in psychological experiments and enhance understanding of cultural differences in psychological functions; 2) to contribute to the scientific research in cognitive and developmental psychology. I cannot guarantee, however, that you will receive any direct benefits from participating in this study.

PAYMENT FOR PARTICIPATION: You will receive a monetary compensation of 10 dollars per hour of participation for your Introductory Psychology course (i.e., if the study takes 1.5 hours to complete, you will receive \$15). You will also have an option for a "walk through" session by completing some or all of the tasks but withdraw your data from the final data set. Compensation will be provided fully to all participants, even though you do not complete the whole study or choose to just "walk through" the session.

CONFIDENTIALITY: Maintaining confidentiality of our participants is important to us. To ensure anonymity and confidentiality, personal identifying information will appear only on the consent form, receipt and background questionnaire. Electronic data and paper-pencil tasks will be labelled only using experiment-specific codes and will be stored in separate locations from the identifiable personal information. Therefore, no connection will be detected between the identifying information and your performance on experimental tasks in any way. Only the primary researcher and assigned research assistant will have access to the collected data. All physical copies of the data will be stored in locked filing cabinets in the Cognitive Aging Lab, and access will be restricted to investigators involved in the study. All electronic data will be encrypted and password-protected. Physical copies of the data will be shredded when no longer required (usually 10 years after the publication of related articles based on the data). Data will be used in future publication, conference presentation, reports, and/or possibly in secondary analyses. In any of these cases, only group results will be reported and no individual identification information will be included.

VOLUNTARY PARTICIPATION AND WITHDRAWAL: Participation in this study is voluntary. You can choose whether to be in this study or not. If you volunteer to be in this study, you may withdraw at any time without consequences of any kind. If you choose to withdraw from this study you may also choose to withdraw your data from the study. You may also choose not to answer any question(s) and still remain in the study. Your choice of whether or not to participate will not influence your future relations with Ryerson University. 
QUESTIONS ABOUT THE STUDY: If you have any questions about the research now, please ask. If you have questions later about the research, you may contact Lingqian Li (Email: lingqian.li@psych.ryerson.ca or Phone: (416) 979-5000, ext.4987)

This study has been reviewed by the Ryerson University Research Ethics Board. If you have questions regarding your rights as a research participant in this study, please contact:

Toni Fletcher, Research Ethics Coordinator

Research Ethics Board

Office of the Vice President, Research and Innovation

Ryerson University

350 Victoria Street

Toronto, Ontario M5B 2K3

416-979-5042 or toni.fletcher@ryerson.ca

INTEREST IN RECEIVING RESULTS OF THE STUDY: If you are interested in learning about the results of this study, please check the box below and provide your contact information (Email address, if you prefer electronic copy; or Full mailing address, if you prefer hard copy). We will provide them to you upon completion of the study. Please be reminded that only group data will be reported and you will not be able to find the information directly linked to you.

No, I am not interested.

Yes, I am interested in receiving results of the study, and I consent to provide contact information to the researcher for this purpose.

Contact (Email or Address):

\section{SIGNATURE OF RESEARCH PARTICIPANT/LEGAL REPRESENTATIVE:}

Your signature below indicates that you have read the information in this agreement and have had a chance to ask any questions you have about the study "Cross Cultural Comparison in Memory Binding" as described herein. Your questions have been answered to your satisfaction, and you agree to participate in this study. You have been given a copy of this form.

Name of Participant (please print)

Signature of Participant

Date

Name of Legal Representative (if applicable) 


\section{Appendix II: Participant Consent Forms (SONA Recruitment)}

\section{RYERSON}

UNIVERSITY

\section{CONSENT TO PARTICIPATE IN RESEARCH Cross Cultural Comparison in Memory Binding (SONA Recruitment)}

You are being invited to participate in a research study. Please read this Consent Form so that you understand what your participation will involve. Before you consent to participate, please ask any questions necessary to be sure you understand what your participation will involve.

INVESTIGATORS: This research study is being conducted by Lingqian Li, Master student, Department of Psychology, under the supervision of Dr. Lixia Yang, associate professor, Department of Psychology, Ryerson University. Results of this study will contribute to a Master's thesis study of the principal investigator. This study is sponsored by Canadian Institutes of Health Research (CIHR).If you have any questions or concerns about this study, please contact Lingqian Li, at lingqian.li@ psych.ryerson.ca, or 416-979-5000, ext.4987.

PURPOSE OF THE STUDY: This study aims to examine how cultural background could affect people's ability to bind information in memory. Thirty students enrolled in the Introduction to Psychology (PSY102/202) courses will be recruited as research participant for this study.

DESCRIPTION OF THE STUDY AND YOUR PARTICIPATION: The study is constituted of one session, which will take approximately one hour to complete. It will take place in the Cognitive Aging Laboratory at the Psychology Research and Training Centre of Ryerson University (105 Bond St., Toronto). Prior to the task, the procedure will be explained and you will have opportunity to ask questions.

If you volunteer to participate in this study, you will be asked to do the following things during the experiment:

- Computerized tasks: you will be required to view and study some pictures, then make responses as instructed by the experimenter.

- Paper-and-pencil tests and questionnaires: you will be asked to complete a set of questions that include queries on: demographic information (e.g., age, gender, and native language), education (e.g., the number of years at each educational institute), and personal health (e.g., illness, vision, hearing problems, any previous head injuries, and current medication use); emotion (e.g., describe your feelings at the moment); and vocabulary.

We request all the above information in order to assess what factors have effects on the tasks we ask you to perform today. You may refuse to answer any particular questions or any tasks without any negative consequence. You will be fully debriefed at the end of the session, any questions you may have about this study will also be answered. If you are interested to know the research findings of this experiment, you can choose to leave your contact information to the experimenter. A copy of the published work can be made available to you upon publication. 
EXPERIMENTAL ASPECTS OF THIS STUDY: None of the procedures or questionnaires used in this study is experimental in nature, in the sense that they have all been used by other researchers and found to be safe and useful. From a procedural point of view, part of this study is considered "experimental" because by following the procedure described above, the study examines the impact of some variables (called the "independent variables") on other variables (called the "dependent variables"). More information about the variables will be provided at the end of the study.

POTENTIAL RISKS AND DISCOMFORTS: There is minimal to no anticipated risk involved in this study. The tests used in this study are standardized psychological tests and the pictures used can be frequently encountered in newspaper, radio, TV, or movies. Every effort will be taken to ensure that you are as comfortable as possible throughout the session. If any of the pictures or questions makes you feel discomfort, you can take a break, choose not to answer a particular question, or discontinue participating, for any reason during the experiment, without receiving any penalties. You will still be rewarded the full credit as stated in the study description.

POTENTIAL BENEFITS TO PARTICIPANTS AND/OR TO SOCIETY: The anticipated benefits of participation in this study include: 1) to gain knowledge in psychological experiments and enhance understanding of cultural differences in psychological functions; 2) to contribute to the scientific research in cognitive and developmental psychology. I cannot guarantee, however, that you will receive any direct benefits from participating in this study.

INCENTIVE FOR PARTICIPATION: You will receive 1\% bonus mark per hour of participation for your Introductory Psychology course. You will also have an option for a "walk through" session by completing some or all of the tasks but withdraw your data from the final data set. Compensation will be provided fully to all participants, even though you do not complete the whole study or choose to just "walk through" the session.

CONFIDENTIALITY: Maintaining confidentiality of our participants is important to us. To ensure anonymity, personal identifying information will appear only on the consent form, receipt and background questionnaire. Electronic data and paper-pencil tasks will be labelled only using experiment-specific codes and will be stored in separate locations from the personal identifiable information. Therefore, no connection will be detected between the identifying information and your performance on experimental tasks in any way. Only the primary researcher and assigned research assistant will have access to the collected data. All physical copies of the data will be stored in locked filing cabinets in the Cognitive Aging Lab, and access will be restricted to investigators involved in the study. All electronic data will be encrypted and password-protected. Physical copies of the data will be shredded when no longer required (usually 10 years after the publication of related articles based on the data). Data will be used in future publication, conference presentation, reports, and/or possibly in secondary analyses. In any of these cases, only group results will be reported and no individual identification information will be included.

VOLUNTARY PARTICIPATION AND WITHDRAWAL: Participation in this study is voluntary. You can choose whether to be in this study or not. If you volunteer to be in this study, you may withdraw at any time without consequences of any kind. If you choose to withdraw from this study you may also choose to withdraw your data from the study. You may also choose not to answer any question(s) and still remain in the study. Your choice of whether or not to participate will not influence your future relations with 
Ryerson University.

QUESTIONS ABOUT THE STUDY: If you have any questions about the research now, please ask. If you have questions later about the research, you may contact Lingqian $\mathrm{Li}$ (Email: lingqian.li@ psych.ryerson.ca or Phone: (416) 979-5000, ext.4987) This study has been reviewed by the Ryerson University Research Ethics Board. If you have questions regarding your rights as a research participant in this study, please contact:

Toni Fletcher, Research Ethics Coordinator

Research Ethics Board

Office of the Vice President, Research and Innovation

Ryerson University

350 Victoria Street

Toronto, Ontario M5B 2K3

416-979-5042 or toni.fletcher@ryerson.ca

INTEREST IN RECEIVING RESULTS OF THE STUDY: If you are interested in learning about the results of this study, please check the box below and provide your contact information (Email address, if you prefer electronic copy; or Full mailing address, if you prefer hard copy). We will provide them to you upon completion of the study. Please be reminded that only group data will be reported and you will not be able to find the information directly linked to you.

$\square$ No, I am not interested.

Yes, I am interested in receiving results of the study, and I consent to provide contact information to the researcher for this purpose.

Contact (Email or Address):

\section{SIGNATURE OF RESEARCH PARTICIPANT/LEGAL REPRESENTATIVE:}

Your signature below indicates that you have read the information in this agreement and have had a chance to ask any questions you have about the study "Cross Cultural Comparison in Memory Binding" as described herein. Your questions have been answered to your satisfaction, and you agree to participate in this study. You have been given a copy of this form.

Name of Participant (please print)

Signature of Participant

Date

Name of Legal Representative (if applicable) 


\section{Appendix III: Participant Debriefing Sheet}

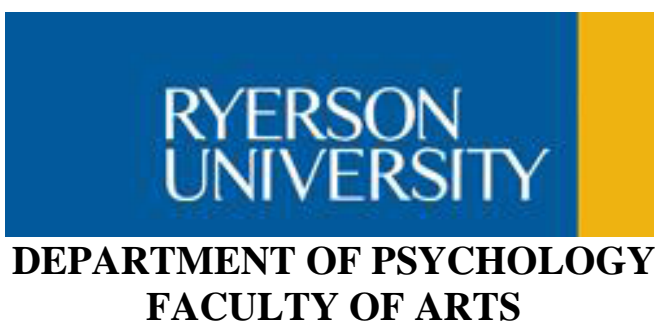

\section{DEBRIEFING SHEET \\ Cross Cultural Comparison in Memory Binding}

Thank you for participating in this experiment!

Converging studies have demonstrated that people from the Eastern and Western cultures process information differently (e.g., Ji, Peng, \& Nisbett, 2000; Kitayama, 2002). In contrast to the analytical thinking Westerners, people from East Asia tend to view the world more holistically and are more sensitive to the context in which information is embedded (Nisbett, Peng, Choi, \& Norenzayan, 2001). A recent study (Yang et al., 2013) found that Chinese participants showed higher context memory (i.e., correctly identify under which context was the item studied) than the Canadian participants when instructed to study items in a meaningful social context. However, since Chinese culture is more collectivist and more oriented towards maintaining interpersonal relationships (Oyserman, Coon, \& Kemmelmeier, 2002), the social context could be more salient to the Chinese participants than Canadian participants. Therefore it remains unclear whether the culture effect observed in Yang et al. was due to active context processing in general, or the result of more salient social context for the Chinese.

In this particular study, I intend to disentangle the effect of social salience from the meaningful context processing, by introducing equally meaningful but non-social contexts (i.e., estimating the height and weight of person from the head shot photographs) to the paradigm. As stated in the consent form, the study examines the impact of the independent variables on the dependent variables. In this study, the independent variables include culture background (Chinese vs. European Canadian) and context condition (Social-context vs. Non-social-context), and the dependent variables include memory for the picture-context link and reaction time. It is hypothesized that (1) Chinese participants will perform better at linking the pictures with the contexts than Canadian participants; and (2) this cultural difference in memory performance will be more prominent under the social context condition compared to the non-social condition.

If you have any questions or concerns regarding this study, please contact the principal investigator, Lingqian Li at lingqian.li@ psych.ryerson.ca, or call (416) 979-5000, extension 4987. I greatly appreciate your time and participation. 
If you have any questions regarding your rights as a human participant in this study, you may contact the Ryerson University Research Ethics Board for information: Toni Fletcher (REB Coordinator) at (416) 979-5042, or email toni.fletcher@ryerson.ca. For more information, you may wish to consult the following sources:

Ji, L. J., Peng, K., \& Nisbett, R. E. (2000). Culture, control, and perception of relationship in the environment. Journal of Personality and Social Psychology, 78, 943-955.

Kitayama, S. (2002). Culture and basic psychological processes: Toward a system view of culture. Psychological Bulletin, 128, 189-196.

Nisbett, R. E., Peng, K., Choi, I., \& Norenzayan, A. (2001). Culture and systems of thought: Holistic vs. analytic cognition. Psychological Review, 108, 291-310.

Oyserman, D, Coon, H. M., Kemmelmeier, M. (2002). Rethinking individualism and collectivism: Evaluation of theoretical assumptions and meta-analyses.

Psychological Bulletin, 128(1), 3-72.

Yang, L., Li, J., Spaniol, J., Hasher, L., Wilkinson, A., Yu, J., Niu, Y. (2013). Aging, culture, and memory for socially meaningful item-context associations: An east-west cross cultural comparison study. PLOS ONE, 8(4), 1-7. doi:10.1371/journal.pone.0060703 


\section{Appendix IV: Photo Difficulty Rating Questionnaire \\ PHOTO DIFFICULTY RATING QUESTIONNAIRE}

Please reflect back to your experiences during the computer tasks. In general, how difficult was it for you to associate the faces with the provided words? Please rate the difficulty level by circling the number on a scale of 1 to 7 , with 1 meaning "not difficult at all" and 7 meaning "very difficult".

\begin{tabular}{|c|c|c|c|c|c|}
\hline 1 & 2 & 3 & 4 & 5 & 6 \\
\hline $\begin{array}{c}\text { Not } \\
\text { difficult at } \\
\text { all }\end{array}$ & & & $\begin{array}{l}\text { Neither } \\
\text { easy nor } \\
\text { difficult }\end{array}$ & & \\
\hline
\end{tabular}

Did you find associating the faces with the words particularly difficult for a specific group of people in the computer tasks?

No, it was the same for all faces.

Yes, it was more difficult for the following groups (please circle the groups that applied to you, you can circle more than one group):
1. Older adult faces
(for friendly/success for height/weight)
2. Younger adult faces
(for friendly/success for height/weight)
3. Female faces
(for friendly/success for height/weight)
4. Male faces
(for friendly/success for height/weight)
5. Western faces
(for friendly/success for height/weight)
6. East Asian faces
(for friendly/success for height/weight)

Did you use any strategy to help you rate the faces during phase 1? If yes, what is it? 


\section{References}

Baltes, P. B. (1993). The aging mind: potential and limits. The Gerontologist, 33(5), 580594. doi:10.1093/geront/33.5.580

Bentin, S., \& Deouell, L. Y. (2000). Structural encoding and identification in face processing: erp evidence for separate mechanisms. Cognitive Neuropsychology, 17(1), 35-55. doi:10.1080/026432900380472

Blais, C., Jack, R. E., Scheepers, C., Fiset, D., \& Caldara, R. (2008). Culture shapes how we look at faces. PloS One, 3(8), e3022. doi:10.1371/journal.pone.0003022

Block, N. (1995). The Mind as the Software of the Brain. In D. N. Osherson, L. Gleitman, S. M. Kosslyn, S. Smith, \& S. Sternberg (Eds.), Thinking: An invitation to the cognitive science (Vol. 3, pp. 377-425). Cambridge, MA: MIT Press. Retrieved from http://www.nyu.edu/gsas/dept/philo/faculty/block/papers/msb.html

Bruce, V., \& Young, A. (1986). Understanding face recognition. British Journal of Psychology, 77(3), 305-327. doi:10.1111/j.2044-8295.1986.tb02199.x

Buchtel, E. E., \& Norenzayan, A. (2009). Thinking across cultures : Implications for dual processes. In J. S. B. T. Evans \& K. Frankish (Eds.), In two minds: Dual processes and beyond (pp. 217-238). Oxford University Press.

doi:10.1093/acprof:oso/9780199230167.003.0010

Caldara, R., Zhou, X., \& Miellet, S. (2010). Putting culture under the "spotlight" reveals universal information use for face recognition. PloS One, 5(3), e9708. doi:10.1371/journal.pone.0009708 
Choi, I., Koo, M., \& Jong An Choi. (2007). Individual differences in analytic versus holistic thinking. Personality \& Social Psychology Bulletin, 33(5), 691-705. doi:10.1177/0146167206298568

Chomsky, N. (2002). Syntactic Structures (2nd ed.). Berlin: Walter de Gruyter GmbH \& Co.

Chua, H. F., Boland, J. E., \& Nisbett, R. E. (2005). Cultural variation in eye movements during scene perception. Proceedings of the National Academy of Sciences of the United States of America, 102(35), 12629-12633. doi:10.1073/pnas.0506162102

Chua, H. F., Chen, W., \& Park, D. C. (2006). Source memory, aging and culture. Gerontology, 52(5), 306-13. doi:10.1159/000094612

Dewhurst, S. a, Hay, D. C., \& Wickham, L. H. V. (2005). Distinctiveness, typicality, and recollective experience in face recognition: a principal components analysis. Psychonomic Bulletin \& Review, 12(6), 1032-1037. doi:10.3758/BF03206439

Goh, J. O. S., Chee, M. W., Tan, J. C., Venkatraman, V., Hebrank, A., Leshikar, E. D., ... Park, D. C. (2007). Age and culture modulate object processing and object-scene binding in the ventral visual area. Cognitive, Affective \& Behavioral Neuroscience, 7(1), 44-52. doi:10.3758/CABN.7.1.44

Gutchess, A. H., Welsh, R. C., Boduroglu, A., \& Park, D. C. (2006). Cultural differences in neural function associated with object processing. Cognitive, Affective \& Behavioral Neuroscience, 6(2), 102-109. doi:10.3758/CABN.6.2.102

Han, S., Northoff, G., Vogeley, K., Wexler, B. E., Kitayama, S., \& Varnum, M. E. W. (2013). A cultural neuroscience approach to the biosocial nature of the human brain. 
Annual Review of Psychology, 64, 335-359.

doi:10.1146/annurev-psych-071112-054629

Hansen, C. (1983). Language and logic in ancient China. University of Michigan Press. Hayward, W. G. (2012). HKU Visual Cognition Laboratory - Face Datbase. Retrieved September 27, 2012, from http://viscog.hku.hk/facedb.htm

Hedden, T., Ketay, S., Aron, A., Markus, H. R., \& Gabrieli, J. D. E. (2008). Cultural influences on neural substrates of attentional control. Psychological Science, 19(1), 12-7. doi:10.1111/j.1467-9280.2008.02038.x

Horne, J. A., \& Ostberg, O. (1976). A self-assessment questionnaire to determine morningness-eveningness in human circadian rhythms. International Journal of Chronobiology, 4, 97-110.

Jenkins, L. J., Yang, Y.-J., Goh, J., Hong, Y.-Y., \& Park, D. C. (2010). Cultural differences in the lateral occipital complex while viewing incongruent scenes. Social Cognitive and Affective Neuroscience, 5(2-3), 236-241. doi:10.1093/scan/nsp056

Ji, L. J., Peng, K., \& Nisbett, R. E. (2000). Culture, control, and perception of relationships in the environment. Journal of Personality and Social Psychology, 78(5), 943-955. doi:10.1037/0022-3514.78.5.943

Kanwisher, N., \& Yovel, G. (2006). The fusiform face area: a cortical region specialized for the perception of faces. Philosophical Transactions of the Royal Society of London. Series B, Biological Sciences, 361(1476), 2109-2128.

doi:10.1098/rstb.2006.1934 
Kelly, D. J., Miellet, S., \& Caldara, R. (2010). Culture shapes eye movements for visually homogeneous objects. Frontiers in Psychology, 1(April), 6. doi:10.3389/fpsyg.2010.00006

Ko, S.-G., Lee, T.-H., Yoon, H.-Y., Kwon, J.-H., \& Mather, M. (2011). How does context affect assessments of facial emotion? The role of culture and age. Psychology and Aging, 26(1), 48-59. doi:10.1037/a0020222

Masuda, T., Ellsworth, P. C., Mesquita, B., Leu, J., Tanida, S., \& Van de Veerdonk, E. (2008). Placing the face in context: cultural differences in the perception of facial emotion. Journal of Personality and Social Psychology, 94(3), 365-381.

doi:10.1037/0022-3514.94.3.365

Masuda, T., \& Nisbett, R. (2001). Attending holistically versus analytically: comparing the context sensitivity of Japanese and Americans. Journal of Personality and Social Psychology, 81(5), 922-934. doi:10.1037/0022-35I4.81.5.922

Masuda, T., \& Nisbett, R. E. (2006). Culture and change blindness. Cognitive Science, 30(2), 381-399. doi:10.1207/s15516709cog0000_63

Meissner, C. a., \& Brigham, J. C. (2001). Thirty years of investigating the own-race bias in memory for faces: A meta-analytic review. Psychology, Public Policy, and Law, 7(1), 3-35. doi:10.1037//1076-8971.7.1.3

Minear, M., \& Park, D. C. (2004). A lifespan database of adult facial stimuli. Behavior Research Methods, Instruments, \& Computers : A Journal of the Psychonomic Society, Inc, 36, 630-633. doi:10.3758/BF03206543 
Miyamoto, Y., Yoshikawa, S., \& Kitayama, S. (2011). Feature and Configuration in Face Processing: Japanese Are More Configural Than Americans. Cognitive Science, 35(3), 563-574. doi:10.1111/j.1551-6709.2010.01163.x

Nisbett, R. E., \& Masuda, T. (2003). Culture and point of view. Proceedings of the National Academy of Sciences of the United States of America, 100(19), 1116311170. doi:10.1073/pnas.1934527100

Nisbett, R. E., \& Miyamoto, Y. (2005). The influence of culture: holistic versus analytic perception. Trends in Cognitive Sciences, 9(10), 467-473. doi:10.1016/j.tics.2005.08.004

Nisbett, R. E., \& Norenzayan, A. (2002). Culture and cognition. In Steven 's Handbook of Experimental Psychology (Third Edit.). Retrieved from http://www.jstor.org/stable/10.2307/2952552

Nisbett, R. E., Peng, K., Choi, I., \& Norenzayan, A. (2001). Culture and systems of thought: holistic versus analytic cognition. Psychological Review, 108(2), 291-310. doi:10.1037/0033-295X.108.2.291

Norenzayan, A., \& Heine, S. J. (2005). Psychological universals: what are they and how can we know? Psychological Bulletin, 131(5), 763-784. doi:10.1037/0033-2909.131.5.763

O’Toole, a J., Deffenbacher, K. a, Valentin, D., \& Abdi, H. (1994). Structural aspects of face recognition and the other-race effect. Memory \& Cognition, 22(2), 208-224. doi:10.3758/BF03208892 
Park, D. C., \& Huang, C.-M. (2010). Culture Wires the Brain: A Cognitive Neuroscience Perspective. Perspectives on Psychological Science : A Journal of the Association for Psychological Science, 5(4), 391-400. doi:10.1177/1745691610374591

Radloff, L. S. (1977). The CES-D Scale: A Self Report Depression Scale for Research in the General. Applied Psychological Measurement, 1, 385-401. doi:10.1177/014662167700100306

Rahhal, T. a, May, C. P., \& Hasher, L. (2002). Truth and character: sources that older adults can remember. Psychological Science, 13(2), 101-105. doi:10.1111/1467-9280.00419

Rhodes, G., Hayward, W. G., \& Winkler, C. (2006). Expert face coding: configural and component coding of own-race and other-race faces. Psychonomic Bulletin \& Review, 13(3), 499-505. doi:10.3758/BF03193876

Richler, J. J., Cheung, O. S., \& Gauthier, I. (2011). Holistic processing predicts face recognition. Psychological Science, 22(4), 464-471. doi:10.1177/0956797611401753

Rowe, G., Hasher, L., \& Turcotte, J. (2009). Age and synchrony effects in visuospatial working memory. Quarterly Journal of Experimental Psychology, 62(10), 18731880. doi:10.1080/17470210902834852

Schultz, R. T. (2005). Developmental deficits in social perception in autism: the role of the amygdala and fusiform face area. International Journal of Developmental Neuroscience, 23(2-3), 125-141. doi:10.1016/j.ijdevneu.2004.12.012 
Singelis, T. M. (1994). The Measurement of Independent and Interdependent Self-Construals. Personality and Social Psychology Bulletin, 20(5), 580-591. doi:10.1177/0146167294205014

Statistics Canada. (2013). 2011 National Household Survey. (Catalogue no. 99-004-XWE). Retrieved September 5, 2014 from Statistics Canada: http://www12.statcan.gc.ca/nhs-enm/2011/dp-pd/prof/index.cfm?Lang=E.

Tanaka, J. W., \& Farah, M. J. (1993). Parts and wholes in face recognition. The Quarterly Journal of Experimental Psychology, 46A(2), 225-245. doi:10.1080/14640749308401045

Tanaka, J. W., \& Farah, M. J. (2003). The holistic representation of faces. In M. A. Peterson \& G. Rhodes (Eds.), Perception of faces, objects, and scenes: analytic and holistic processes (pp. 53-74). Oxford: Oxford University Press. Retrieved from http://journals.lww.com/jneuro-ophthalmology/Abstract/2006/06000/Perception_of_ Faces,_Objects,_and_Scenes_Analytic.18.aspx

Tanaka, J. W., Kiefer, M., \& Bukach, C. M. (2004). A holistic account of the own-race effect in face recognition: evidence from a cross-cultural study. Cognition, 93(1), B1-9. doi:10.1016/j.cognition.2003.09.011

Todorov, A., Mende-Siedlecki, P., \& Dotsch, R. (2013). Social judgments from faces. Current Opinion in Neurobiology, 23(3), 373 - 380. doi:10.1016/j.conb.2012.12.010

Triandis, H. C. (1995). Individualism and collectivism. Calorado: Westview Press.

Wang, R., Li, J., Fang, H., Tian, M., \& Liu, J. (2012). Individual differences in holistic processing predict face recognition ability. Psychological Science, 23(2), 169-177. doi:10.1177/0956797611420575 
Watson, D., Clark, L. A., \& Tellegen, A. (1988). Development and validation of brief measures of positive and negative affect: the PANAS scales. Journal of Personality and Social Psychology, 54(6), 1063-1070. doi:10.1037/0022-3514.54.6.1063

Wechsler, D. (1997). Wechsler Adult Intelligence Scale. San Antonio, TX: Psychological Corporation.

Yama, H., Nishioka, M., Horishita, T., Kawasaki, Y., \& Taniguchi, J. (2007). A dual process model for cultural differences in thought. Mind \& Society, 6(2), 143-172. doi:10.1007/s11299-007-0028-4

Yang, L., Chen, W., Ng, A. H., \& Fu, X. (2013). Aging, culture, and memory for categorically processed information. The Journals of Gerontology. Series B, Psychological Sciences and Social Sciences, 68(6), 872-881. doi:10.1093/geronb/gbt006

Yang, L., Li, J., Spaniol, J., Hasher, L., Wilkinson, A. J., Yu, J., \& Niu, Y. (2013). Aging, culture, and memory for socially meaningful item-context associations: an East-West cross-cultural comparison study. PloS One, 8(4), e60703. doi:10.1371/journal.pone.0060703

Young, S. G., Hugenberg, K., Bernstein, M. J., \& Sacco, D. F. (2012). Perception and motivation in face recognition: a critical review of theories of the Cross-Race Effect. Personality and Social Psychology Review, 16(2), 116-142.

doi:10.1177/1088868311418987 